\title{
Gene flow analysis method, the D-statistic, is robust in a wide parameter space
}

\author{
Yichen Zheng ${ }^{*}$ (D) and Axel Janke
}

\begin{abstract}
Background: We evaluated the sensitivity of the D-statistic, a parsimony-like method widely used to detect gene flow between closely related species. This method has been applied to a variety of taxa with a wide range of divergence times. However, its parameter space and thus its applicability to a wide taxonomic range has not been systematically studied. Divergence time, population size, time of gene flow, distance of outgroup and number of loci were examined in a sensitivity analysis.

Result: The sensitivity study shows that the primary determinant of the D-statistic is the relative population size, i.e. the population size scaled by the number of generations since divergence. This is consistent with the fact that the main confounding factor in gene flow detection is incomplete lineage sorting by diluting the signal. The sensitivity of the D-statistic is also affected by the direction of gene flow, size and number of loci. In addition, we examined the ability of the f-statistics, $\hat{f}_{G}$ and $\hat{f}_{\text {hom, }}$ to estimate the fraction of a genome affected by gene flow; while these statistics are difficult to implement to practical questions in biology due to lack of knowledge of when the gene flow happened, they can be used to compare datasets with identical or similar demographic background.

Conclusions: The D-statistic, as a method to detect gene flow, is robust against a wide range of genetic distances (divergence times) but it is sensitive to population size. The D-statistic should only be applied with critical reservation to taxa where population sizes are large relative to branch lengths in generations.
\end{abstract}

Keywords: Gene flow, The D-statistic, Sensitivity, Population size, Parameter space, Simulation

\section{Background}

Traditional phylogenetic analyses that assume a bifurcating tree fails to model complicated evolutionary processes such as incomplete lineage sorting (ILS), gene flow, and horizontal gene transfer [1]. Gene flow, or introgression, refers to alleles from one species entering a different (and usually closely related) species through migration and hybridization. It is a violation of the assumption in traditional phylogenetics that speciation is a sudden event and no exchange of genetic information occurs thereafter. Incomplete lineage sorting refers to an occurrence where lineages of a certain locus fail to coalesce in the branch directly in the past of their population divergence, resulting in three or more un-coalesced lineages existing in a population $[1,2]$. This can result in discordance between the genealogy of that locus (gene

*Correspondence: yzheng2@uni-koeln.de

Biodiversität und Klima Forschungszentrum, Senckenberg Gesellschaft für Naturforschung, 60325 Frankfurt, Germany tree) and population split history (species tree). These factors caused phylogenetics to enter an era of multilocus analysis and is facilitated by availability of wholegenome sequencing [3]. There are multiple methods designed to reconstruct a "species tree," a tree that describes speciation processes as splitting of populations [4-7]. However, these methods still aim for a completely bifurcating tree. To fully resolve the complexity during speciation and divergence, one would need to treat "phylogenetic incongruence [as] a signal, rather than a problem" [8].

Analysis of gene flow must take ILS into account, because both processes generate gene trees that are incongruent with the species tree. Among the earliest methods to detect gene flow are a homoplasy-based analysis that finds taxa that are intermediate between putative parent species [9], and a gene tree comparison that identifies locus divergence younger than the species' divergence [10]. Later methods can be generally separated into two categories: likelihood-based/Bayesian- 
and parsimony-based, using different interpretations of the coalescent models. Likelihood or Bayesian methods, such as Phylonet [11, 12] and CoalHMM [13] are based on a priori evolutionary models, and are applicable across a large range of conditions. However, their disadvantages often include excessive computation times and the need to estimate a large number of parameters and to specify priors that are difficult to obtain accurately, but which can crucially affect the outcome.

The D-statistic, also known as the ABBA-BABA statistic, is a useful and widely applied parsimony-like method for detecting gene flow despite the existence of ILS [14, 15]. This method is designed to be used on either one of two types of data: (1) sequence alignment where there is only one or a few samples per taxa, or (2) SNP data where the frequency of each allele in each population is known. This method compares the number of ABBA and BABA sites parsimony-informative sites that support a different phylogeny than the species tree, and determine whether they are statistically equal in number. The two genealogies discordant with the species tree, $\mathrm{ABBA}$ and $\mathrm{BABA}$ are equally likely to be produced by ILS; therefore they should not differ in number if only ILS, but not gene flow is present. A significant difference between ABBA and BABA sites indicates that two non-sister species are more similar to each other than expected, which is interpreted as a signal of gene flow. The D-statistic has been used in numerous studies to detect gene flow between closely related species of bears [16], equids [17], butterflies [18] as well as hominids [14], and plants $[19,20]$, and even microbial pathogens [21].

The D-statistic (see Methods for formula) is used for a group of four taxa with an established phylogeny (Fig. 1) to detect gene flow between two ingroups that are not sister species (in this case, $\mathrm{H} 2$ and $\mathrm{H} 3$ ). The value of $\mathrm{D}$ is affected by a number of parameters; a) fraction of gene flow (f), b) divergence times, c) time of gene flow

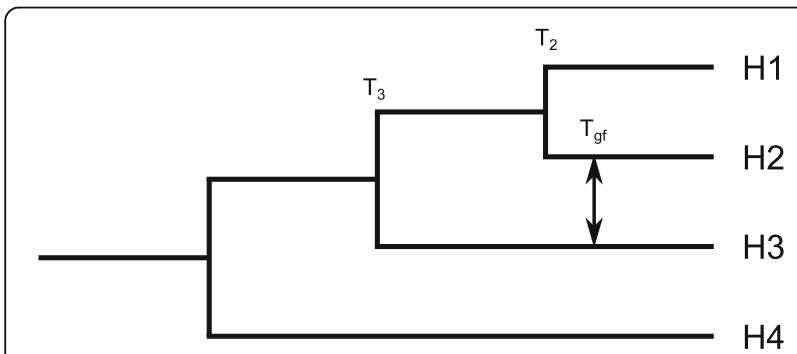

Fig. 1 A four-taxon tree required to implement the D-statistic. The four taxa are designated as $\mathrm{H} 1, \mathrm{H} 2, \mathrm{H} 3$ and $\mathrm{H} 4$, with $\mathrm{H} 4$ serving as the outgroup. Gene flow between $\mathrm{H} 2$ and $\mathrm{H} 3$ (shown with arrows) or $\mathrm{H} 1$ and $\mathrm{H} 3$ can be detected with the D-statistic. $\mathrm{T}_{3}, \mathrm{~T}_{2}$ and $\mathrm{T}_{\text {gf }}$ denotes the time passed since each event. and d) population size. The "fraction of gene flow" refers to the fraction of recipient genome that descended from the donor population. The value of $\mathrm{f}$ cannot exceed 0.5 , otherwise the source of gene flow will contribute more to the recipient's genome than its lineage described in the species tree. As a result, the species tree would need to be changed to represent the lineages that provide the majority of the genome. Given the above parameters, the expected value of $\mathrm{D}$ is (formula from [15]):

$$
E(D)=\frac{3 f\left(T_{3}-T_{g f}\right)}{3 f\left(T_{3}-T_{g f}\right)+4 N(1-f)\left(1-\frac{1}{2 N}\right)^{T_{3}-T_{2}}+4 N f\left(1-\frac{1}{2 N}\right)^{T_{3}-T_{g f}}}
$$

Here $\mathrm{f}$ is the fraction of gene flow, $\mathrm{N}$ is the population size, $T_{3}$ is the divergence time between the donor and recipient of gene flow, $T_{2}$ is the divergence time between the recipient of gene flow and its sister species that have not received gene flow, and $T_{g f}$ is the time of the gene flow event. All times are in units of generations. The expected value of $\mathrm{D}$ does not have a linear or mathematically simple relationship with the fraction of gene flow. Therefore, the calculation of $\mathrm{f}$ from $\mathrm{D}$ is impossible without knowing the divergence times, time of gene flow, and population size with high accuracy [22]. As a result, the D-statistic is often used as a qualitative measure where a significant $\mathrm{D}$ indicates presence of gene flow. Furthermore, the D-statistic can be highly susceptible to random variation in short sequences, making it unfit for detecting which regions have been affected by gene flow [22].

Durand et al. [15] proposed an alternative measure, $\hat{f}_{G}$ (see Methods for formula), which is expected to have a linear relationship with the actual fraction of gene flow, $\mathrm{f}$, and is unaffected by population size. This is based on an assumption that a locus that underwent $100 \%$ gene flow will convert $\mathrm{H} 2$ into a member of the $\mathrm{H} 3$ population. Martin et al. [22] developed two additional estimators of $\mathrm{f}, \hat{f}_{\text {Hom }}$ and $\hat{f}_{d}$. $\hat{f}_{\text {Hom }}$ (see Materials and Methods for formula) uses the sequences of $\mathrm{H} 3$ as a control to determine how much of H2's genome is affected by gene flow (see Materials and Methods), under an assumption that as the gene flow increases, $\mathrm{H} 2$ and $\mathrm{H} 3$ will be completely homogenized (which is only correct if the gene flow is extremely recent). $\hat{f}_{d}$ compares $\mathrm{H} 2$ and $\mathrm{H} 3$ in a site-by-site basis and choose a "donor population" in which the derived allele has a higher frequency (therefore requiring population-level data), thus being able to explicitly model gene flow for both directions $\mathrm{H} 2->\mathrm{H} 3$ and H3 - > H2. Martin et al. [22] showed that both $\hat{f}_{G}$ and $\hat{f}_{\text {Hom }}$ have a high variance among loci and occasionally had a value above 1 , indicating that they are subject 
to higher stochasticity; on the other hand, $\hat{f}_{d}$ performs in a more stable way.

However, little is known about the parameter space in which the D- and f-statistics can be reliably used, which is of particular interest to biologists. The D-statistic is commonly used on species that diverged recently or have small genetic distances; it was originally developed to test hybridization between humans and Neanderthals, which diverged about 270,000-440,000 years ago (some 20,000 generations), and have a DNA sequence distance of $0.3 \%$ [14]. On the other hand, the method has been applied to species groups such as butterflies Heliconius timareta and H. melpomene [18], which were estimated to have diverged two million years ago with a DNA sequence distance of more than 1\% [23]. This corresponds to 8 to 24 million generations, given a generation time estimate between one and three months [24, 25]. To date the maximal sequence divergence on which the D- and f-statistics have been applied is 4 to $5 \%$, in mosquitoes of the genus Anopheles [26] and plants of the genus Mimulus [27]. It is still unknown if the D-statistic will be less effective on taxa that are highly diverged; an intuitive prediction would be the deterioration of the $\mathrm{D}$ statistic's effectiveness with increasingly divergent taxa, due to signals being overwhelmed by noise such as multiple substitutions and even saturation.

In the original simulation tests [15], the times of divergence and gene flow were not varied, and all polymorphic sites were independent without linkage. In the simulation tests by Martin et al. [22], the divergence times were strictly proportional to population size, not allowing variation of one without the other. The probability of two lineages ( $\mathrm{H} 1$ and $\mathrm{H} 2$ ) coalescing on the branch leading to their divergence is determined by the ratio of branch length (in generations) and population size $[28,29]$. If they fail to coalesce within the branch, a third lineage (H3) will appear in the population, leading to ILS, which produces two alternative gene trees that lead to ABBA and BABA sites at a same rate. The ratio of population size to divergence time, being a direct determinant of frequency of incongruent gene trees [1], is expected to have an effect on the sensitivity of the Dand f-statistics, i.e. how likely a gene flow event can be detected given that it exists. We predict that the Dstatistic is less sensitive, and the f-statistics are less robust, in datasets with a higher population size relative to divergence time.

Therefore, we raise the question whether the effectiveness of the D- and f-statistics are affected by variation of divergence and gene flow time as well as population size, particularly when the ratio between population size and time scale is varied. In addition, we analyzed the statistical significance of the D-statistic instead of the statistic itself, particularly its sensitivity, because it is better suited as a qualitative measure. Finally, we will analyze the effect of gene flow direction and locus size on the statistical significance of the D-statistic, and the interaction between these variables (in particular, divergence times and gene flow direction). We are convinced that this will provide a valuable guide for future geneticists to better judge limits of incorrect interpretation of the $\mathrm{D}$ - and f-statistics as a method to detect and measure gene flow.

\section{Methods}

\section{Definition of the $D$ and f-statistics}

According to the notions used by $[15,22]$ we review the parameters and their definitions used in the D and $\mathrm{f}$ statistics for this study. Assume aligned or mapped DNA sequences are sampled from an asymmetric phylogeny of four taxa, (((H1, H2), H3), H4). $N_{A B B A}(H 1, H 2, H 3$, H4) is defined as the number of nucleotide sites in which $\mathrm{H} 2$ and $\mathrm{H} 3$ share an allele, while $\mathrm{H} 1$ and $\mathrm{H} 4$ share a different allele. Similarly, $N_{B A B A}(H 1, H 2, H 3, H 4)$ is the number of nucleotide sites in which $\mathrm{H} 1$ and $\mathrm{H} 3$ share an allele, while $\mathrm{H} 2$ and $\mathrm{H} 4$ share a different allele. These numbers can refer to either one locus or the entire genome. The D-statistic is denoted as:

$$
D(H 1, H 2, H 3, H 4)=\frac{N_{A B B A}(H 1, H 2, H 3, H 4)-N_{B A B A}(H 1, H 2, H 3, H 4)}{N_{A B B A}(H 1, H 2, H 3, H 4)+N_{B A B A}(H 1, H 2, H 3, H 4)}
$$

The numerator of this formula is represented by $S(H 1$, $H 2, H 3, H 4)$.

In addition to the D-statistic, we examined two fstatistics that can be calculated without requiring the allele frequency in populations. These statistics, $\hat{f}_{G}$ and $\hat{f}_{\text {hom }}$, are estimators of $\mathrm{f}$, the fraction of gene flow. While they utilize four taxa with the same tree as the Dstatistic, $\hat{f}_{G}$ has an additional requirement that at least two samples must be collected from the H3 population. The f-statistics are calculated as:

$$
\begin{gathered}
\hat{f}_{G}=\frac{S(H 1, H 2, H 3, H 4)}{S(H 1, H 3 a, H 3 b, H 4)} \\
\hat{f}_{\text {hom }}=\frac{S(H 1, H 2, H 3, H 4)}{S(H 1, H 3, H 3, H 4)}
\end{gathered}
$$

$\mathrm{H} 3 \mathrm{a}$ and H3b are two samples from the H3 lineage, assuming to be two unrelated individuals in the same population. The $\mathrm{H} 3$ used in the calculation of $\hat{f}_{G}$ can be either H3a or H3b. For $\hat{f}_{\text {hom }}, \mathrm{H} 3$ is used twice in the denominator; $N_{B A B A}(H 1, H 3, H 3, H 4)$ is always zero, because $\mathrm{H} 3$ cannot be different from itself, so $S(H 1, H 3$, $H 3, H 4)$ is identical to $N_{A B B A}(H 1, H 3, H 3, H 4)$, i.e., alleles shared by $\mathrm{H} 1$ and $\mathrm{H} 4$ but not by $\mathrm{H} 3$.

Tests of significance for the D- and f-statistics were done with a jackknife method, in which $5 \mathrm{Mb}$ blocks 
were removed one at a time to estimate a standard error that is approximately normally distributed [30, 31].

\section{Simulating of species trees, gene trees and DNA sequences}

We used coalescence models to simulate gene trees from species trees, in order to take in account ILS in addition of gene flow. A species tree with fixed topology (Fig. 2) was used as the basis of the simulation, in which $\mathrm{T}_{\mathrm{gf}}, \mathrm{T}_{2}$, $\mathrm{T}_{3}$ and $\mathrm{T}_{4}$ are independent variables we control in input. Of note is that $\mathrm{H} 3 \mathrm{a}$ and $\mathrm{H} 3 \mathrm{~b}$ represent two samples from the same population, used to calculate $\hat{f}_{G}$. H2f and $\mathrm{H} 3 \mathrm{f}$ are used as lineages introduced by gene flow, that originates from $\mathrm{H} 2$ and $\mathrm{H} 3$ respectively. The parameters were set according to Table 1 (Scheme 1), producing 27 species trees with different branch lengths. Note that both branch length and population size were scaled with the reciprocal of substitution rate, $1 / \mu$, so that the results would be applicable to organisms with a wide range of substitution rates. Along a branch with the length $\mathrm{T}=\mathrm{k} \times 1 / \mu$ generations, $\mathrm{k}$ substitutions per nucleotide are expected.

SimPhy [32] was used to simulate gene trees from species trees, using a coalescence-based Wright-Fisher model [33, 34]. The population size, $\mathrm{Ne}$, is constant throughout the tree and proportional to divergence level (Table 1, Scheme 1). Gene trees were produced from each species tree; 15 sets of 50,000 gene trees were produced, which include three replicates for each of the five population sizes. In each gene tree, a sample of each lineage $(\mathrm{H} 1, \mathrm{H} 2, \mathrm{H} 2 \mathrm{f}, \mathrm{H} 3 \mathrm{a}, \mathrm{H} 3 \mathrm{~b}, \mathrm{H} 3 \mathrm{f}$ and $\mathrm{H} 4)$ was taken and the divergence times between samples were

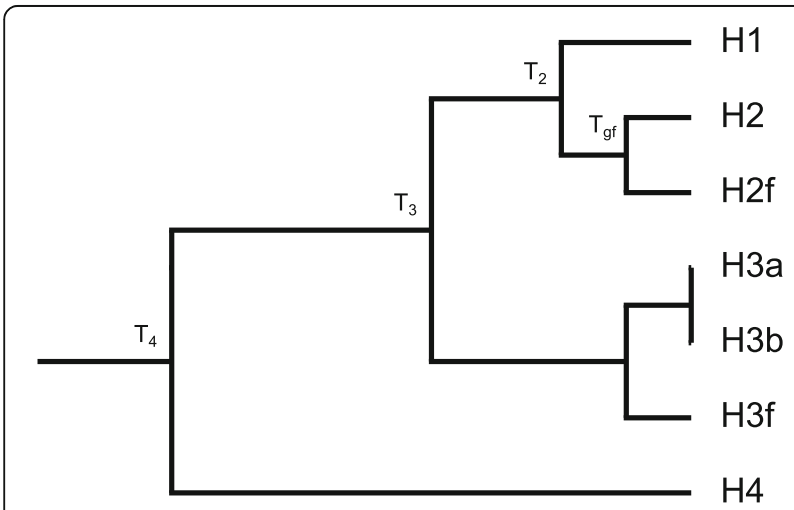

Fig. 2 The species tree used for the coalescent-based gene tree simulation. $T_{g f}, T_{2}, T_{3}$ and $T_{4}$ are respectively divergence or gene flow times of the corresponding event, measured in the unit of generations. $\mathrm{H} 3 \mathrm{a}$ and $\mathrm{H} 3 \mathrm{~b}$ represent two independent samples from the same $\mathrm{H} 3$ population. $\mathrm{H} 2 \mathrm{f}$ represents an introgressed lineage originating from the $\mathrm{H} 2$ population, and similarly $\mathrm{H} 3 \mathrm{f}$ represents an introgressed lineage originating from the $\mathrm{H} 3$ population simulated as constrained by the species tree, i.e. divergence times between populations. The resulting gene tree may have a different topology than the species tree. We denote the ratio $\mathrm{Ne} / \mathrm{T}_{3}$ as the "relative population size." A total of 135 parameter combinations and 405 datasets were generated. All other parameters were set to default.

The branch lengths in the simulated gene trees were then converted from units of generations to units of substitutions per nucleotide, during which the parameter 1/ $\mu$ was cancelled out. The program INDELible [35] was used to simulate non-coding DNA sequences from gene trees. A 20-kb-long locus was simulated from each gene tree. The sequence evolution model was HKY with a transition/transversion ratio of 3.6 [36, 37], gamma distribution of substitution rate with shape factor $\alpha=1$, and a GC content of $40 \%$. Each of the 135 parameter combinations produced 50,000 unlinked loci, with a total size of $1 \mathrm{~Gb}$. A typical mammalian genome is $3 \mathrm{~Gb}$ and contains about a half repeat sequences; thus, $1 \mathrm{~Gb}$ is close to the size of a mammalian genome alignment with repeats and difficult-to-map regions (such as centromeres and telomeres) excluded.

ABBA and BABA site counts for D, the $\hat{f}_{G}$ and $\hat{f}_{\text {hom }}$ statistics were calculated in each locus, under three alternative situations: (1) under no gene flow, H1, H2, $\mathrm{H} 3 \mathrm{a}$ and $\mathrm{H} 4$ are used as the four sampled sequences, and $\mathrm{H} 3 \mathrm{a}$ and $\mathrm{H} 3 \mathrm{~b}$ are used as two samples of $\mathrm{H} 3$ to calculate $\hat{f}_{G}$; (2) under gene flow from $\mathrm{H} 3$ to $\mathrm{H} 2$. Here $\mathrm{H} 1$, $\mathrm{H} 3 \mathrm{f}, \mathrm{H} 3 \mathrm{a}$ and $\mathrm{H} 4$ are used as the four sampled sequences, and $\mathrm{H} 3 \mathrm{a}$ and $\mathrm{H} 3 \mathrm{~b}$ are used as two samples of $\mathrm{H} 3$ to calculate $\hat{f}_{G}$; (3) under gene flow from $\mathrm{H} 2$ to $\mathrm{H} 3$, $\mathrm{H} 1, \mathrm{H} 2, \mathrm{H} 2 \mathrm{f}$ and H4 are used as the four sampled sequences. Calculation of $\hat{f}_{G}$ in (3), as it requires sampling two individuals in the gene flow recipient, is deemed to be beyond the scope of this study. The reason is that when two samples of $\mathrm{H} 3$ (recipient of gene flow) are taken, it is possible that only one sample is introgressed in a certain locus; however this possibility is dependent on whether the introgressed allele is fixed, which requires a more complicated coalescence model.

Hereafter, an "introgression test" refers to the following procedures: given a fraction of gene flow of a certain direction, $\mathrm{f}(0 \leq \mathrm{f} \leq 0.5)$, in a $1 \mathrm{~Gb}$ dataset, $50,000 \times \mathrm{f}$ loci are randomly chosen to be under gene flow, while the other $50,000 \times(1-f)$ loci are not under gene flow. Using this combination, the $\mathrm{D}, \hat{f}_{G}$ and $\hat{f}_{\text {hom }}$ statistics are calculated using the formulae detailed above and tested using the jackknife method, where every 250 loci (5Mbp) are used as one block [14]. A test is significant if the resulting $\mathrm{Z}$ score (the value of $\mathrm{D}$-statistic divided by its standard error) is above 3, a value chosen for strong significance based on $[14,38]$ corresponding to $p<0.0013$. 
Table 1 Variables and constant parameters used in the study

\begin{tabular}{|c|c|c|c|c|}
\hline Variable & $\begin{array}{l}\text { Scheme 1: analysis of branch I } \\
\text { engths and population }\end{array}$ & $\begin{array}{l}\text { Scheme 2: analysis of } \\
\text { outgroup distance }\end{array}$ & $\begin{array}{l}\text { Scheme 3: analysis of } \\
\text { number and size of loci }\end{array}$ & $\begin{array}{l}\text { Scheme 4: analysis } \\
\text { of diploid data }\end{array}$ \\
\hline Divergence $\left(T_{3}\right)$ & $0.001,0.01$ or $0.1 \times 1 / \mu$ Generations & 0.001 or $0.01 \times 1 / \mu$ Generations & 0.001 or $0.01 \times 1 / \mu$ Generations & $\begin{array}{l}0.001 \text { or } 0.01 \times 1 / \mu \\
\text { Generations }\end{array}$ \\
\hline $\mathrm{T}_{\mathrm{g} f} \mathrm{f} \mathrm{T}_{2}$ & $0.25,0.5$ or 0.75 & 0.5 & 0.5 & \multirow{2}{*}{$\begin{array}{l}\text { One of these combinations: } \\
0.25 \text { and } 0.1 ; 0.5 \text { and } 0.5 ; \\
\text { or } 0.75 \text { and } 0.9 \text {. }\end{array}$} \\
\hline $\mathrm{T}_{2} / \mathrm{T}_{3}$ & $0.1,0.5$ or 0.9 & 0.5 & 0.5 & \\
\hline$T_{4} / T_{3}$ & 2 & $1.5,2,5,10$ or 20 & 2 & 2 \\
\hline Population size & $0.2,0.5,1,2$ or $5 T_{3}$ & $0.2,0.5,1,2$ or $5 T_{3}$ & $0.2,0.5,1,2$ or $5 T_{3}$ & $0.2,1$, or $5 T_{3}$ \\
\hline Number of loci & 50,000 & 50,000 & $\begin{array}{l}2000,5000,10,000,20,000 \\
50,000 \text { or } 100,000\end{array}$ & 50,000 \\
\hline Replicates & 3 & 3 & 3 & 3 \\
\hline Total datasets & 405 & 150 & 180 & 54 \\
\hline
\end{tabular}

Datasets were simulated in three schemes, focusing on different parameters. Parameters not varied in that scheme are in italics

The $\mathrm{Z}$ score of the $\mathrm{D}, \hat{f}_{G}$ and $\hat{f}_{\text {hom }}$ statistics are calculated separately, therefore, their significance are also determined separate from each other. In summary, an introgression test is a test for the D- and f-statistics and their significance, given the fraction of gene flow, $\mathrm{f}$, and the dataset.

\section{Sensitivity test}

A sensitivity test is an analysis on parameters that would cause false negatives in a test. In our case, the sensitivity test is a power analysis; determining the power of the Dstatistic to detect gene flow. Sensitivity tests were conducted in two steps. In the first step, f values of $0,0.001$, $0.002, \ldots, 0.009,0.01,0.015,0.02,0.03, \ldots, 0.09,0.1,0.15$, $0.2,0.3,0.4$, and 0.5 (hereafter called the "basic f list") were used for introgression tests. Each $f$ value other than 0 was tested 3 times. The smallest $f$ for which all 3 times tested positive was denoted $\mathrm{f}_{0}$; the number two places before $\mathrm{f}_{0}$ in the "basic $\mathrm{f}$ list" was denoted $\mathrm{f}_{\min }$ (if $\mathrm{f}_{0}=$ 0.001 or $0.002, f_{\min }=0.001$ ), and the number immediately after $\mathrm{f}_{0}$ was denoted $\mathrm{f}_{\max }$ (if $\mathrm{f}_{0}=0.5, \mathrm{f}_{\max }=0.5$ ). In the second step, $f$ values between $f_{\min }$ and $f_{\max }$ were tested with an interval of 0.001 . Each $f$ value was tested 500 times. Using a logistic regression, the smallest $f$ that have an $80 \%$ probability to produce a significant result was used as the threshold value to indicate sensitivity, as standard for power analyses [39]. This threshold is called MF80, (Minimal Fraction for $80 \%$ significance), and lower MF80 indicates better sensitivity. If the predicted probability of the D-statistic being significant is still less than $80 \%$ when $\mathrm{f}=0.5$, the D-statistic is not usable in this dataset. In this situation, MF80 is set as 0.501 for the downstream statistical analysis rather than treating it as missing data, so that we can make use of the knowledge that the D-statistic is extremely insensitive in this dataset. It will only cause underestimation of the correlations between sensitivity and parameters as the true MF80 (had we allow $\mathrm{f}>0.5$ ) will be at least 0.501 .

The $\hat{f}_{G}$ and $\hat{f}_{\text {hom }}$ statistics were linearly regressed with the input $f$ using the data from the entire "basic $f$ list"; the slope of this regression is used as estimate of $\hat{f}_{G} / \mathrm{f}$ and $\hat{f}_{\text {hom }} / \mathrm{f}$.

\section{Analyzing the effect of outgroup distance}

In this section, we studied how the genetic distance between outgroup ( $\mathrm{H} 4)$ and ingroups (H1-H3) affect the sensitivity of the D- and f-statistics, given an otherwise identical species tree. The variables used in this section are described in Table 1 (Scheme 2). Of note is that the highest level of divergence is not included because it is least realistic, and the $T_{4} / T_{3}$ ratio is the main variable under study. From each parameter combination, three replicates each of 50,000 gene trees were simulated, and from each gene tree, $20 \mathrm{~kb}$ of non-coding DNA sequences were simulated, using the same method as the previous section. A total of 150 datasets were produced. Analysis of sensitivity of the D- and f-statistics are also conducted using the same methods as the previous section.

\section{Analyzing the effect of number and size of independent loci}

In this section, we studied the impact on the D- and $\mathrm{f}$ statistics by the number of independent loci, given the same species tree and total sequence length. The variables used in this sections are described in Table 1 (Scheme 3). Of note is that the highest level of divergence is removed, and the locus number is the main variable under study. Under a constant total sequence length of $1 \mathrm{~Gb}$, the lengths of each locus under each value are $500 \mathrm{~kb}, 200 \mathrm{~kb}, 100 \mathrm{~kb}, 50 \mathrm{~kb}, 20 \mathrm{~kb}$ and $10 \mathrm{~kb}$. From each parameter combination, three replicate 
datasets were simulated, producing 180 datasets in total. Analysis of the D- and f-statistics were conducted using the same methods as in previous sections.

\section{Robustness of f-statistics}

This section describes an analysis on the robustness of the f-statistics against random variation caused by locus sampling. We used data from 18 parameter combinations in Simulation Scheme 1: $\mathrm{T}_{3}=1 \times 10^{4}$ or $1 \times 10^{5}$ Generations; Population size $=0.2,1$ or $5 \mathrm{~T}_{3} ; \mathrm{T}_{\mathrm{gf}} / \mathrm{T}_{2}$ and $\mathrm{T}_{2} / \mathrm{T}_{3}$ are one of these combinations: 0.25 and $0.1,0.5$ and 0.5 , or 0.75 and 0.9 .

The f-statistics we examined are $\hat{f}_{G}$ and $\hat{f}_{\text {hom }}$ in H3 - > H2 gene flow, and $\hat{f}_{\text {hom }}$ in $\mathrm{H} 2$-> H3 gene flow. For each real $f$ value on the "basic $f$ list" (see above section "Sensitivity Test") we estimated 500 replicate sets of the f-statistics. In each replicate, 50,000 loci are randomly selected from the combined pool of 150,000 loci of the three replicates of that parameter combination (Table 1); within which, $f \times 50,000$ of them are under gene flow and $(1-f) \times 50,000$ are not under gene flow. The $f$ statistics were calculated and their confidence intervals were determined as (statistic $\pm 2 \times$ standard deviation) [15]. In a small number of replications, the jackknife variance of $\hat{f}_{G}$ was calculated as negative (the variance is based on a weighted measure where the weight of a jackknife block is based on the denominator of the fstatistic, which can be negative in some blocks for $\hat{f}_{G}$, because the formula includes a subtraction); in these cases the confidence intervals were treated as missing data.

Pairwise comparisons were conducted in these procedures: Let $i$ and $j$ be real $f$ values from the "basic $f$ list", where $\mathrm{i} \leq \mathrm{j}$. Compare each of the 500 replicate $\hat{f}_{G}$ values where the real $\mathrm{f}$ is i $\left(\hat{f}_{G}(i)\right)$, and each of the 500 replicate $\hat{f}_{G}$ values where the real $\mathrm{f}$ is $\mathrm{j}\left(\hat{f}_{G}(j)\right)$; in the $500 \times 500=$ 250,000 comparisons, record the proportion of comparisons where $\hat{f}_{G}(i)$ is numerically smaller than $\hat{f}_{G}(j)$, and where $\hat{f}_{G}(i)$ is significantly smaller than $\hat{f}_{G}(j)$; in the case where $\mathrm{i}=\mathrm{j}$, record the proportion of comparisons where $\hat{f}_{G}(i)$ is not significantly different from $\hat{f}_{G}(j)$. Significant difference is defined by non-overlapping confidence intervals. The same procedures were also used to compare $\hat{f}_{\text {hom }}$ from gene flow of both directions. The recorded proportions are estimates of the probability that the difference between real $\mathrm{f}$ values (or lack thereof) were correctly identified using the f-statistic.

\section{Diploid data}

To study whether our findings are applicable to diploid data, we simulated additional datasets. The variables used in this section are described in Table 1 (Scheme 4), and the 18 parameter combinations are a subset of the ones from Scheme 1: $\mathrm{T}_{3}=1 \times 10^{4}$ or $1 \times 10^{5}$ Generations; Population size $=0.2,1$ or $5 \mathrm{~T}_{3} ; \mathrm{T}_{\mathrm{gf}} / \mathrm{T}_{2}$ and $\mathrm{T}_{2} / \mathrm{T}_{3}$ are one of these combinations: 0.25 and $0.1,0.5$ and 0.5 , or 0.75 and 0.9 . Gene trees and sequences were simulated using the same procedures as in previous schemes, except that we specified two sequences were sampled from each taxon. One combination of parameters $\left(\mathrm{T}_{3}=1 \times\right.$ $10^{5}$ Generations, Population size $=5 \mathrm{~T}_{3}, \mathrm{~T}_{\mathrm{gf}} / \mathrm{T}_{2}=0.5, \mathrm{~T}_{2} /$ $\mathrm{T}_{3}=0.5$ ) had an additional replication simulated, because one of the original replications resulted in a false positive $(\mathrm{Z}>3$ when no gene flow is present) and was discarded.

Analysis of sensitivity of the D-statistic were conducted using similar methods as the previous sections with special consideration taken for diploid data. During the introgression tests, two methods were used to draw the loci under gene flow for the recipient taxon. In the "same loci" method, the same $50,000 \times \mathrm{f}$ loci are randomly chosen to be under gene flow for both genome copies; in the "random loci" method, two independent sets of 50,000 $\times \mathrm{f}$ loci (allowing overlap) are chosen for the two genome copies. Sites that are heterozygous in any analyzed taxon were excluded from the ABBA and BABA site counts.

\section{Results}

Sensitivity of the D-statistic in relation with divergence time, branch lengths, population size and direction of gene flow

Sensitivity of the D-statistic is described with the minimal fraction of gene flow to have an over $80 \%$ probability producing a significant $(Z>3)$ test result. We call this value MF80 (Minimal Fraction for $80 \%$ significance), and lower MF80 indicates better sensitivity. Figure 3 shows the relationship between four parameters and MF80. Our simulations show that, counterintuitively, MF80 has only a marginal negative correlation with divergence time $(r=-0.146, p=0.003$; for $\log$ MF80 and $\log$ divergence time, $r=-0.105, p=0.034$ ), which indicates a (slightly) better sensitivity in high divergence datasets. MF80 does not change markedly even with large divergences (sequence differences) (Fig. 3a), where $\mathrm{H} 1 / \mathrm{H} 2$ and $\mathrm{H} 3$ have a genetic distance of over $20 \%$. For comparison, mouse and rat have a sequence difference of $15-17 \%$ [40].

On the other hand, MF80 is affected by the population size (Fig. 3b, $r=0.151, p=0.002$ ), indicating better sensitivity with small populations. The correlation between $\log$ population size and $\log$ MF80 is stronger $(r=0.361$, $p<0.0001$ ); this is because population sizes were varied on a logarithmic scale, making the numbers crowd on the lower side when not log-transformed. The strongest 

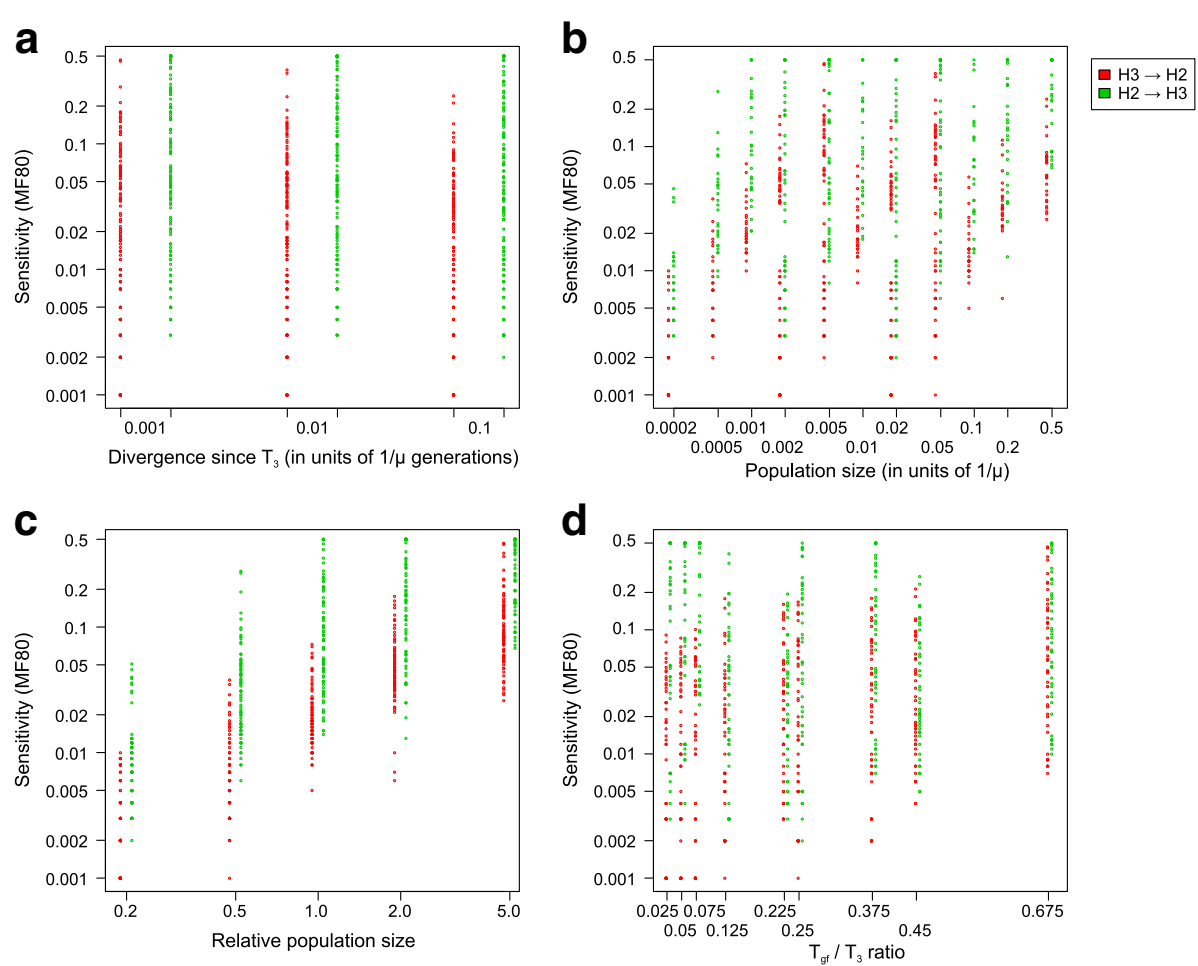

Fig. 3 Sensitivity and input parameters. The relationship of sensitivity as measured with MF80, the minimal fraction of gene flow that produces over $80 \%$ significant D-statistics, and various input parameters: a Divergence, measured in generations between H3's divergence and current time $\left(T_{3}\right)$; b Population size; $\mathbf{c}$ Relative population size, the ratio of population size and divergence generations; $\mathbf{d}$ Relative time of gene flow, the ratio of time of gene flow $\left(T_{\mathrm{gf}}\right)$ and $\mathrm{T}_{3}$. Red points represent gene flow from $\mathrm{H} 3$ to $\mathrm{H} 2$, and green points represent gene flow from $\mathrm{H} 2$ to $\mathrm{H} 3$; the colors are slightly offset on the $x$-axis to ease reading

signal, however, occurs when we compare MF80 with relative population size (Fig. 3c). Relative population size is defined as the ratio of population size and $\mathrm{T}_{3}$, which is number of generations passed since $\mathrm{H} 1, \mathrm{H} 2$ and $\mathrm{H} 3$ split in the species tree. For example, human and Neanderthal have a divergence time of 20,000 generations and an effective population size of about 10,000 , so the relative population size is estimated as $10,000 /$ $20,000=0.5$ [14]. The correlation between MF80 and relative population is $r=0.693(p<0.0001)$, and increases to $r=0.890(\mathrm{p}<0.0001)$ if both are logarithmically transformed. Within each divergence category $(0.001,0.01$ or $0.1 \times 1 / \mu$ Generations), the pattern of correlation is same as for the entire combined dataset.

Finally, there is a weak correlation between the $\mathrm{T}_{\mathrm{gf}} / \mathrm{T}_{3}$ ratio and MF80 (Fig. 3d, $r=0.371, p<0.0001$; with log MF80, $r=0.349, p<0.0001)$, indicating that gene flow events that are more recent are easier to detect. From the correlation analyses, it can be concluded that the sensitivity of the D-statistic is primarily determined by relative population size, and secondly determined by time of gene flow; indeed, these two variables can largely predict the output MF80 under a simple linear (Fig. 4a) or log-linear (Fig. 4b) model, with the latter being more accurate.

Gene flow from $\mathrm{H} 2$ to $\mathrm{H} 3$ was also simulated with the same methods, and the D-statistic's sensitivities were measured as MF80 in all datasets. Regardless of divergence, population size or relative time of gene flow, the D-statistic is less or at most equally sensitive compared to gene flow from $\mathrm{H} 3$ to $\mathrm{H} 2$ (Fig. 5).

Correlations between input parameters to MF80 on the H2-> H3 direction were calculated with the same methods. Similar to the H3- $>$ H2 direction, MF80 is not affected by the divergence (Fig. 3a, $r=-0.098, p=0.048$; if both $\log$ transformed, $r=-0.090, p=0.070$ ), weakly by absolute population size (Fig. 3b, $r=0.239, p<0.0001$; if both log transformed, $r=0.342, p<0.0001$ ), but strongly by relative population size (Fig. 3c, $r=0.826, p<0.0001$; if both $\log$ transformed, $r=-0.090, p<0.0001)$. However, the correlation between MF80 and the $\mathrm{T}_{\mathrm{gf}} /$ $\mathrm{T}_{3}$ ratio is $r=-0.234$ (Fig. $3 \mathrm{~d}, p<0.0001$ ), meaning that younger gene flow events are more difficult to detect than older ones, a counterintuitive finding. The correlation becomes weaker if $\log (\mathrm{MF} 80)$ is used instead $(r=-0.130, p=0.009)$. Further investigation 

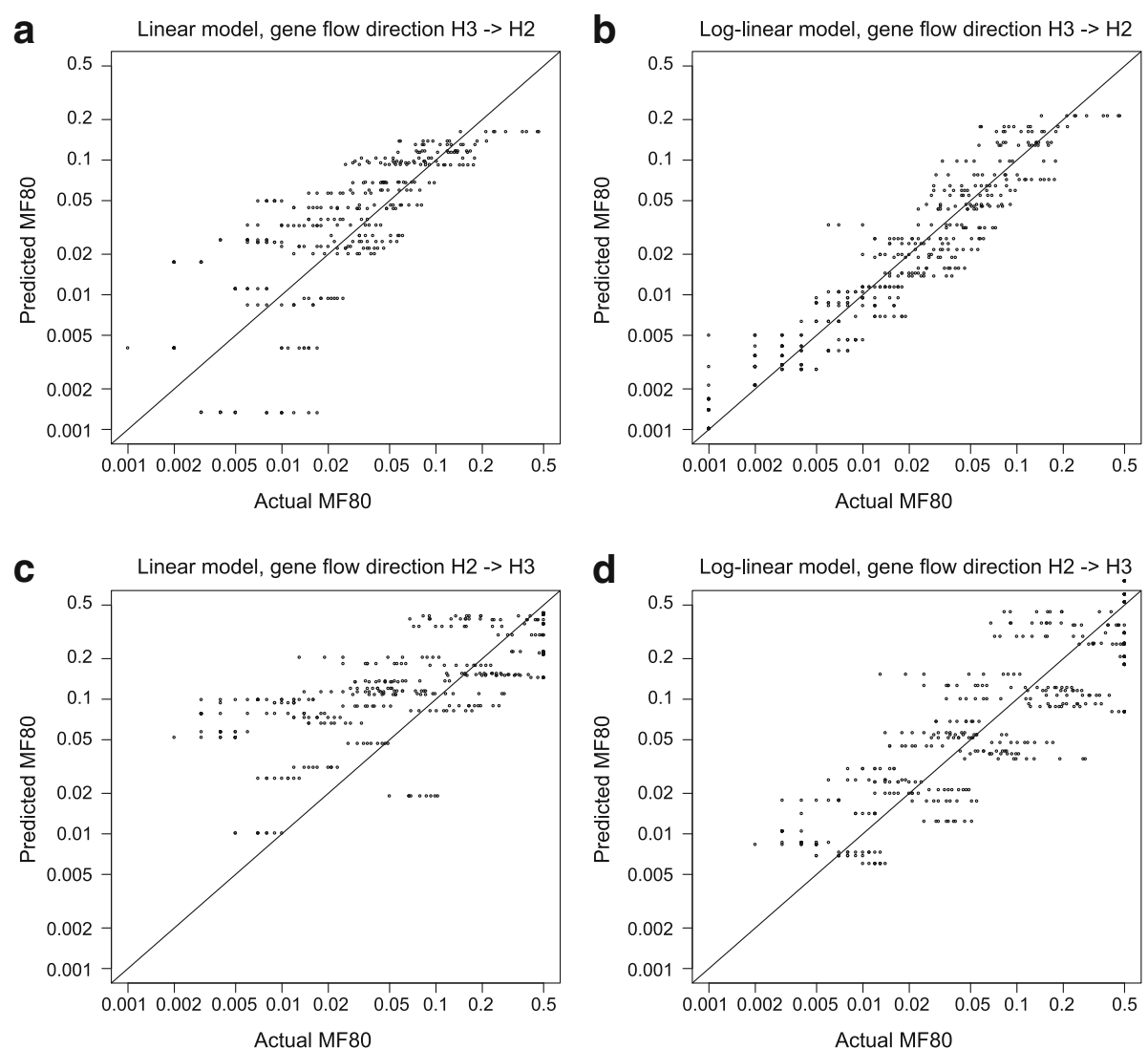

Fig. 4 Comparison of measured and predicted sensitivity. Comparison between sensitivity as measured with MF80 measured from our analysis and MF80 predicted with a linear $(\mathbf{a}, \mathbf{c})$ or log-linear $(\mathbf{b}, \mathbf{d})$ model based on the relative population size and $T_{\mathrm{gf}} / T_{3}$ ratio. $\mathbf{a}, \mathbf{b}$ Direction of gene flow is $\mathrm{H} 3->\mathrm{H} 2$; $\mathbf{c}$, $\mathbf{d}$ Direction of gene flow is $\mathrm{H} 2->\mathrm{H} 3$. The sloped line indicates when the measured and predicted MF80 are equal

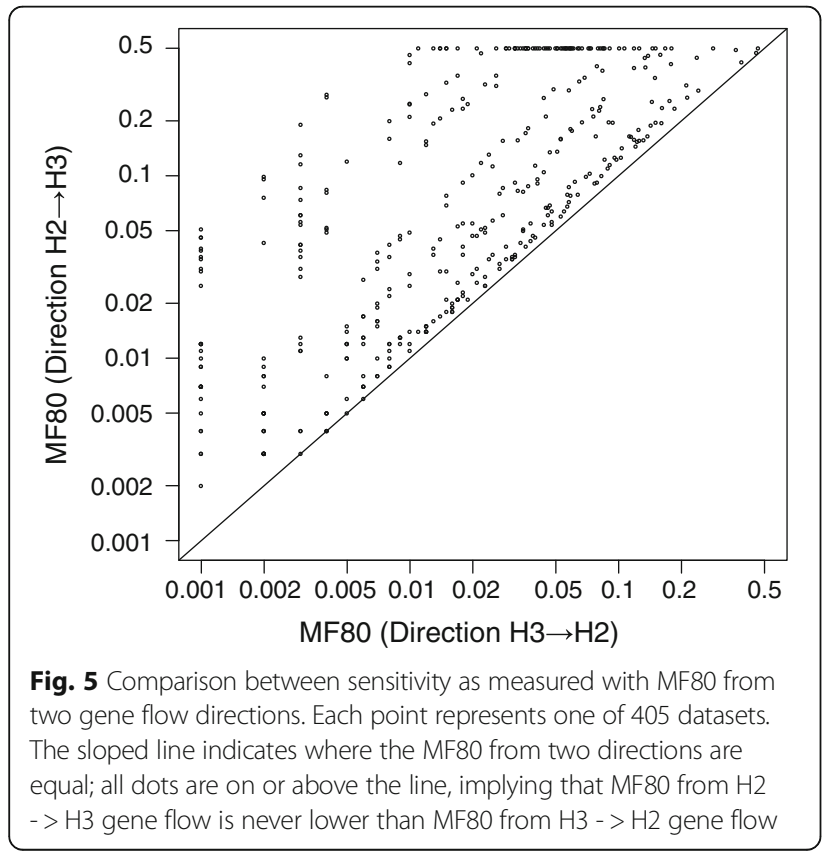

(Additional file 1) showed that MF80 is positively correlated with $\mathrm{T}_{\mathrm{gf}} / \mathrm{T}_{2}$ ratio $(r=0.235, p<0.0001)$, but strongly and negatively correlated with $\mathrm{T}_{2} / \mathrm{T}_{3}$ ratio $(r=-0.440, p<0.0001)$. This pattern is not found in the $\mathrm{H} 3$ - > H2 direction. When $\mathrm{H} 1$ and $\mathrm{H} 2$ diverged later (relative to $\mathrm{H} 3$ divergence time), i.e., $\mathrm{T}_{2} / \mathrm{T}_{3}$ ratio is lower, there are more shared alleles between $\mathrm{H} 1$ and (un-introgressed) H2. Under $\mathrm{H} 3->\mathrm{H} 2$ gene flow, these shared alleles become different, producing more ABBA sites. Under H2 - > H3 gene flow, on the other hand, these shared alleles become shared by all $\mathrm{H} 1, \mathrm{H} 2, \mathrm{H} 3$, producing BBBA patterns, and thus not counted. The ability of MF80 prediction by relative population size and $\mathrm{T}_{\mathrm{gf}} / \mathrm{T}_{3}$ ratio is weaker than the $\mathrm{H} 3$ - > H2 direction (Fig. 4c, d).

For detailed MF80 on both directions for each dataset, see Additional file 2.

\section{Sensitivity of the D-statistic in relation with the distance of outgroup and number of loci}

An intuitive expectation would be that the test's sensitivity decreases when the outgroup (H4) is more distant, as 
a distant outgroup reduces the information quality and amount. Here we tested the effect of the outgroup distance, described by $T_{4} / T_{3}$ ratio, in a range from 1.5 to 20. In comparison, the ratio is about 7.9 times in the earliest usage of the D-stat where $\mathrm{H} 3$ is Neanderthal and H4 is chimpanzee ([14]; human-Neanderthal mean sequence divergence estimated as 825,000 years and human-chimpanzee as 6.5 million years). Figure 6 shows the sensitivity of the D-statistic, measured with MF80, under different $\mathrm{T}_{4} / \mathrm{T}_{3}$ ratio (x-axis) and relative population size (color). It is evident that MF80 is primarily determined by relative population size, but it is unaffected by the $\mathrm{T}_{4} / \mathrm{T}_{3}$ ratio. The correlation between $\mathrm{T}_{4} / \mathrm{T}_{3}$ ratio and MF80 is calculated to be $r=-0.078(p=$ $0.342)$, or $r=-0.021$ ( $p=0.795)$ if log transformed. However, the interaction between $\mathrm{T}_{4} / \mathrm{T}_{3}$ ratio and relative population size is significant $(p=0.005)$. Therefore, we showed that the D-statistic is robust regarding the genetic distance between ingroups and the outgroup.

In each of the above datasets, $1 \mathrm{~Gb}$ of DNA sequences were simulated as 50,000 unlinked loci each of $20 \mathrm{~kb}$. Here we analyzed the effect of locus number and size on sensitivity under a constant total sequence length of $1 \mathrm{~Gb}$. Figure 7 shows the sensitivity of the D-statistic, measured with MF80, for different numbers of loci ( $\mathrm{x}$ axis) and different relative population sizes (color). While the effect of the number of loci is not as strong as that of the relative population size, there is a trend that MF80 becomes smaller (more sensitive) in datasets with shorter sequences of each locus, but increasing number of loci. The correlation between locus number and

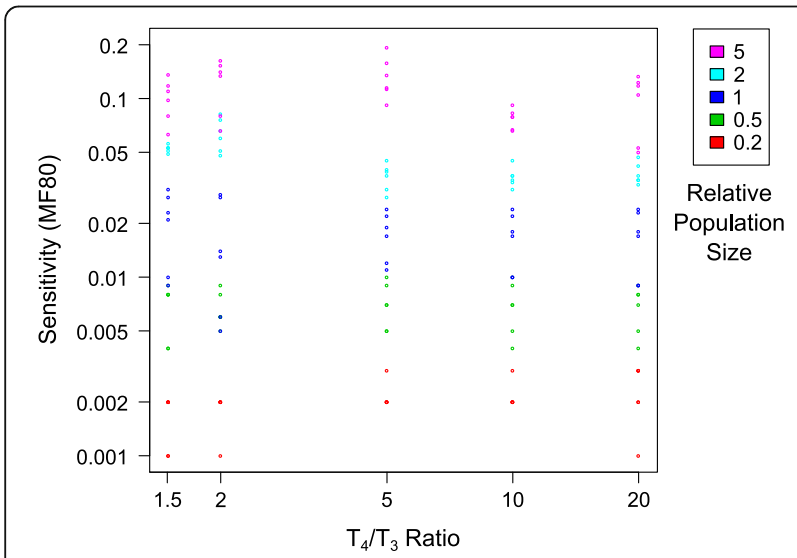

Fig. 6 Sensitivity and distance of outgroup. The relationship between $\mathrm{T}_{4} / T_{3}$ ratio ( $x$-axis), and sensitivity as measured with MF80 (y-axis). A higher $T_{4} / T_{3}$ ratio indicates that the outgroup is more distant to the ingroups, relative to the distance among the ingroups. Colors represent results from analyses for different relative population sizes, with red being the smallest and purple the largest. The analyses show that MF80 is positively and strongly correlated to the relative population size, while MF80 is not notably affected by the $T_{4} / T_{3}$ ratio, either as a whole or within each relative population size

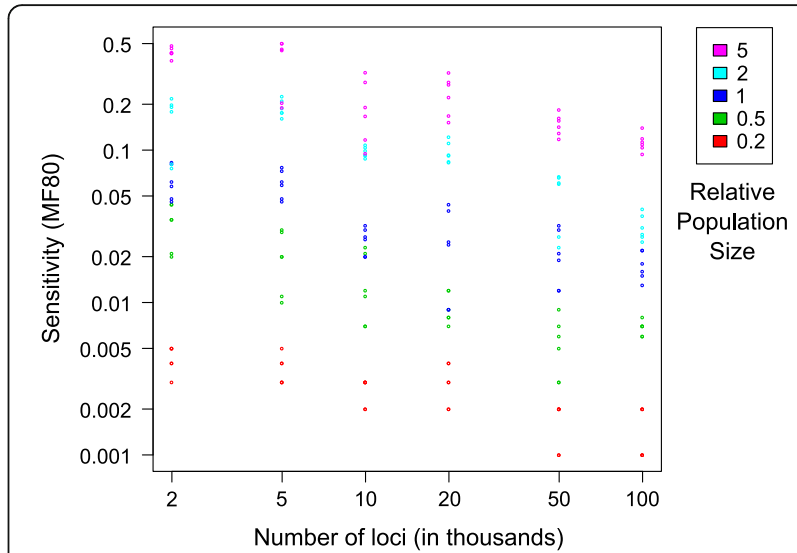

Fig. 7 Sensitivity and number of independent loci. The relationship between the number of independent loci in each 1Gb dataset ( $x$-axis), and sensitivity as measured with MF80 (y-axis). Colors represent results from analyses for different relative population sizes, with red being the smallest and purple the largest; MF80 is positively and strongly correlated with relative population size. MF80 is also correlated with number of loci, with a larger number of loci (thus smaller loci) resulting in lower MF80. The correlation between loci number and MF80 is weaker than the correlation between relative population size and MF80

MF80 is $r=-0.273 \quad(p=0.0002)$, or $r=-0.297 \quad(p<$ $0.0001)$ when both are $\log$ transformed. The interaction between locus number and relative population size is not significant $(p=0.534)$ when both are transformed.

\section{The D-statistic when no gene flow is present}

One potential source of error in this study comes from the difference between $\mathrm{ABBA}$ and $\mathrm{BABA}$ site numbers even when no gene flow is occurring, due to the sampling error during gene tree and sequence simulation. For example, one would expect the MF80 to be underestimated if the zero-f dataset has a positive D-statistic, or vice versa. We used the Z-score of the $D$-statistic when $f=0$ as an indicator of such bias. None of the 405 datasets have a significant Z-score $(|Z|>3$ ) when $f=0$ (which would constitute a false positive). This Z-score is significantly correlated with MF80 (Fig. 8) in the H3-> H2 direction, the correlation is $r=-0.143(p=0.004)$, but not in the H2- $>\mathrm{H} 3$ direction, where $r=-0.089(p=0.072)$. This indicates that the sensitivity is indeed affected by random sampling error, albeit only weakly so. However, we argue that this random noise is canceled out when all 135 datasets are used and it does not bias our general findings. The absolute value $\mathrm{Z}$-score when $\mathrm{f}=0$ is not correlated with most input parameters $(p=0.926$ for divergence, $p=0.076$ for relative population size, and $p=0.056$ for $\mathrm{T}_{\mathrm{gf}} / \mathrm{T}_{3}$ ratio). For the individual Z-scores when $f=0$ in each dataset, see Additional file 2 . 


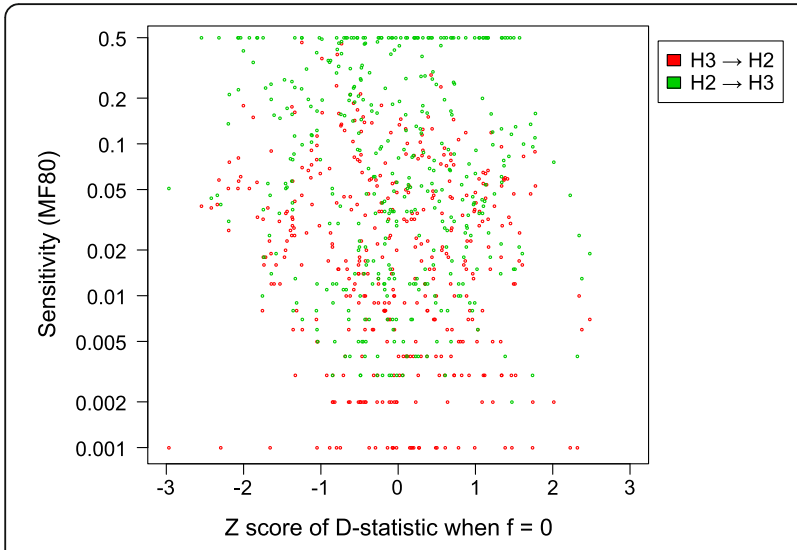

Fig. 8 Z-score for the D-statistic under no gene flow. The relationship between the Z-score of the D-statistics under $f=0$ ( $x$-axis), and sensitivity as measured with MF80 (y-axis). Red points represent gene flow from $\mathrm{H} 3$ to $\mathrm{H} 2$, and green points represent gene flow from $\mathrm{H} 2$ to $\mathrm{H} 3$. The $\mathrm{Z}$-score of the D-statistics under $\mathrm{f}=0$ is expected to be zero; any deviation is caused by random sampling error of loci (noise). There is a weak correlation between MF80 and Z-score of the D-statistics under $\mathrm{f}=0$, indicating that measured sensitivity is slightly influenced by sampling error of loci

\section{Usage and robustness of the f-statistics}

In addition to the D-statistic, we tested the $\hat{f}_{G}$ and $\hat{f}_{\text {hom }}$ statistics, which are estimates of $\mathrm{f}$, the fraction of genome affected by the gene flow event; they were proposed because the D-statistic is qualitative and cannot be used to estimate how strong the gene flow is. In each of the 405 datasets, $\hat{f}_{G}$ and $\hat{f}_{\text {hom }}$ are both linearly correlated to $\mathrm{f}$ where the gene flow direction is H3-> H2, with correlation coefficient $\mathrm{r}$ no smaller than 0.98 in any dataset. The ratios $\hat{f}_{G} / \mathrm{f}$ and $\hat{f}_{\text {hom }} / \mathrm{f}$ are calculated with linear regression models; the estimated parameters of the models can be found in Additional file 2. As expected from [15], $\hat{f}_{G} / \mathrm{f}$ is roughly equal to $1-\frac{T_{G F}}{T_{3}}$. On the other hand, the ratio $\hat{f}_{\text {hom }}$ /f can be most closely estimated as $\left(1-\frac{T_{G F}}{T_{3}}\right) /\left(1+\frac{N_{e}}{T_{3}}\right)$ (See Additional file 2 for the predictors' precision).

The intercept of the linear regression between $\hat{f}_{G}$ (or $\hat{f}_{\text {hom }}$ ) and $\mathrm{f}$ indicates an error, where the f-statistics are non-zero even without actual gene flow. In some datasets with low to medium divergence and large population sizes, $\left|\hat{f}_{G}\right|$ can be above 0.05 even when $\mathrm{f}=0$, meaning that there will be a false positive of gene flow if it is used solely as a predictor of $\mathrm{f}$. All 17 datasets where $\left|\hat{f}_{G}\right|$ $>0.03$ have divergence of 0.001 or $0.01 \times 1 / \mu$ generations and a relative population size of 5 . On the other hand, $\left|\hat{f}_{\text {hom }}\right|$ when $\mathrm{f}=0$ does not exceed 0.01 in all datasets, indicating that it is more robust against false positives compared to $\hat{f}_{G}$.
Significance of $\hat{f}_{\text {hom }}$ can be tested in a similar way to the D-statistic, using jackknife subsampling. Indeed, the sensitivity of $\hat{f}_{\text {hom }}$ is almost identical to D; the MF80, minimal fraction of gene flow for $80 \%$ chance of significance $(Z \geq 3)$, are equal or close to equal in all datasets. On the other hand, $\hat{f}_{G}$ is much more difficult to evaluate statistically. The main reason is that the jackknife makes use of the denominator in each block to determine the weight of each block in the entire dataset; the denominator of $\hat{f}_{G}$ is the difference between two non-zero site counts, which can be negative or even zero, rendering the jackknife algorithm inapplicable.

$\hat{f}_{G}$ under $\mathrm{H} 2$ - > H3 gene flow was not calculated, because our model cannot predict whether the same introgressed loci are fixed for multiple samples in the recipient population. For most datasets, $\hat{f}_{\text {hom }}$ is linearly correlated with $\mathrm{f}$, similar to the $\mathrm{H} 3->\mathrm{H} 2$ direction. However, the correlation is very weak in datasets with low $\mathrm{T}_{2} / \mathrm{T}_{3}$ ratio (very recent divergence between $\mathrm{H} 1$ and $\mathrm{H} 2$ ) and high relative population size, indicating that $\mathrm{f}$ cannot be predicted with $\hat{f}_{\text {hom }}$ even if all parameters are known. The slope of linear regression, $\hat{f}_{\text {hom }} / \mathrm{f}$ can be estimated as $\left(\frac{T_{2}}{T_{3}}-\frac{T_{G F}}{T_{3}}\right) /\left(1+\frac{N_{e}}{T_{3}}\right)$ (See Additional file 2 for the predictor's precision). This ratio is always smaller than what it could be if the direction of gene flow is $\mathrm{H} 3->\mathrm{H} 2$; the difference is stronger when $\mathrm{T}_{2} / \mathrm{T}_{3}$ ratio is low.

Figure 9 shows the difference between the estimated f-statistics from randomly drawn loci and the expected number calculated from the above formulae. The variation of the estimated f-statistics is insensitive to the value of real $\mathrm{f}$. Given the same divergence and introgression times and the same relative population size, $\hat{f}_{G}$ has a larger margin of error than $\hat{f}_{\text {hom }}$, while $\hat{f}_{\text {hom }}$ for both gene flow directions have similar error (Fig. 9a, b, c). The variance of the f-statistics also increases with relative population size (Fig. 9b, d, e for $\hat{f}_{\text {hom }}$ in $\mathrm{H} 3$-> $\mathrm{H} 2$; for $\hat{f}_{\text {hom }}$ in $\mathrm{H} 2->\mathrm{H} 3$ and $\hat{f}_{G}$ the result is similar). There is a slight bias for $\hat{f}_{\text {hom }}$ when the real $\mathrm{f}$ is above 0.2 , towards a lower value for $\mathrm{H} 3->\mathrm{H} 2$ gene flow and a higher value for $\mathrm{H} 2$ - > H3 gene flow (Fig. 9b,c).

However, the expected value of the f-statistic is smaller when the real $\mathrm{f}$ is smaller, which means the relative error can be large in such cases (Fig. 10). Although in extreme cases with large relative population size and low real $f$ the mean error can be over 10 times the expected value (Fig. 10c), such gene flow events lie outside of the Dstatistic's sensitivity and would not be qualitatively detected at the first place. Generally, the f-statistics can be estimated within $\pm 20 \%$ for gene flow events that can be detected, given that population size and divergence and introgression times are known. 


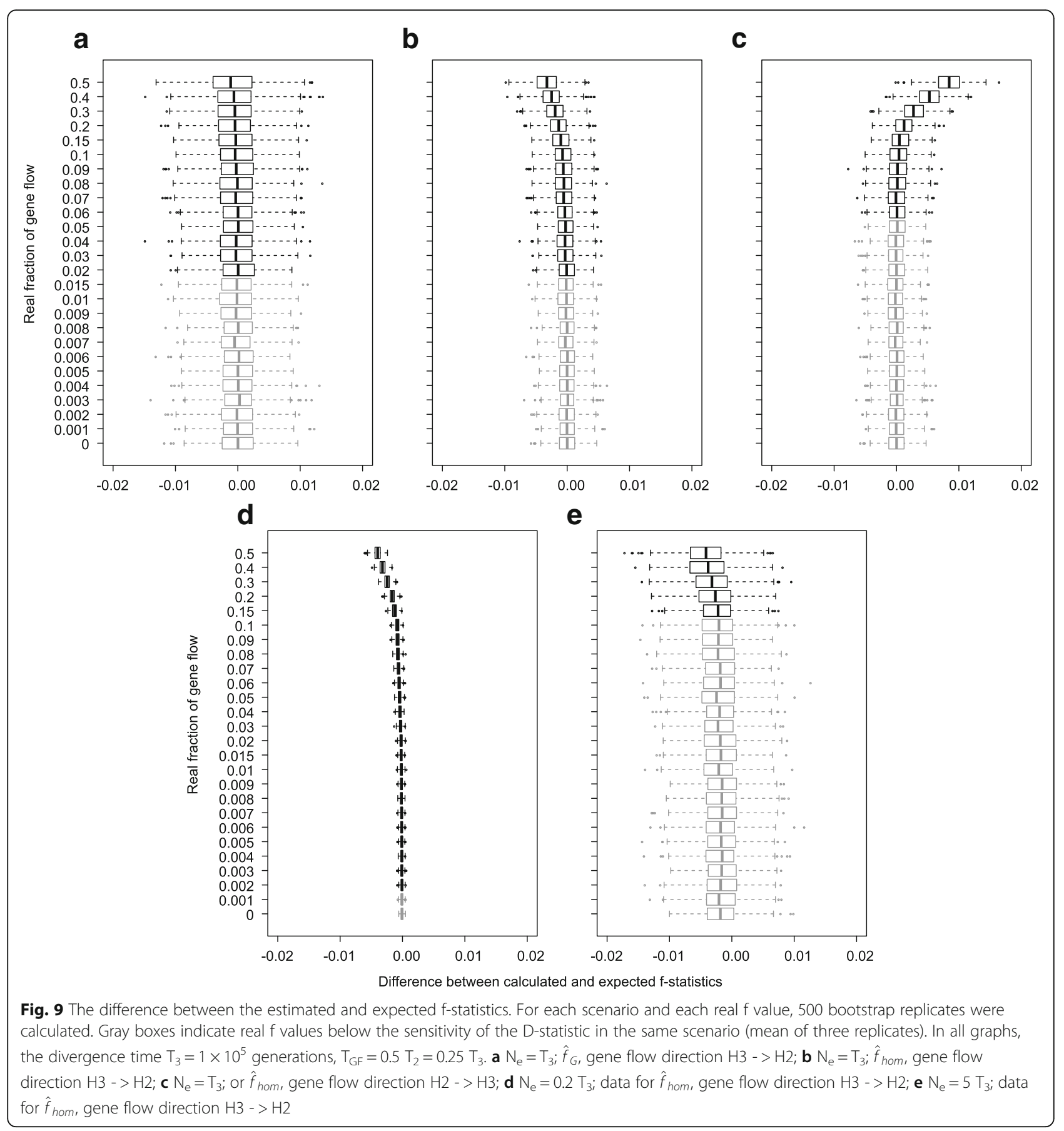

Pairwise comparisons showed similar trends (Fig. 11). When the real $f$ are equal, almost all (>99\%) of comparisons showed no significant difference regardless of demographic scenario, indicating that the false negative rate of f-statistic comparison is very low (circles on the diagonals).

Relative population size is the main factor in determining whether comparisons between two f-statistics correctly identify the relationship of the real $f$, either by numerical comparison or statistical significance. Two $f$ values both above the sensitivity of the $\mathrm{D}$-statistic can be confidently correctly compared by numerical f-statistic comparison, even if statistical significance can be lacking with small differences between the $\mathrm{f}$ values. While $\hat{f}_{G}$ (Fig. 11a, d, g) and $\hat{f}_{\text {hom }}$ (Fig. 11b, e, h) for H3 - > H2 gene flow shows the same level of robustness, $\hat{f}_{\text {hom }}$ for $\mathrm{H} 2->$ H3 (Fig. 11c, f, i) is clearly more difficult to estimate and to compare, under the same divergence and demographic 


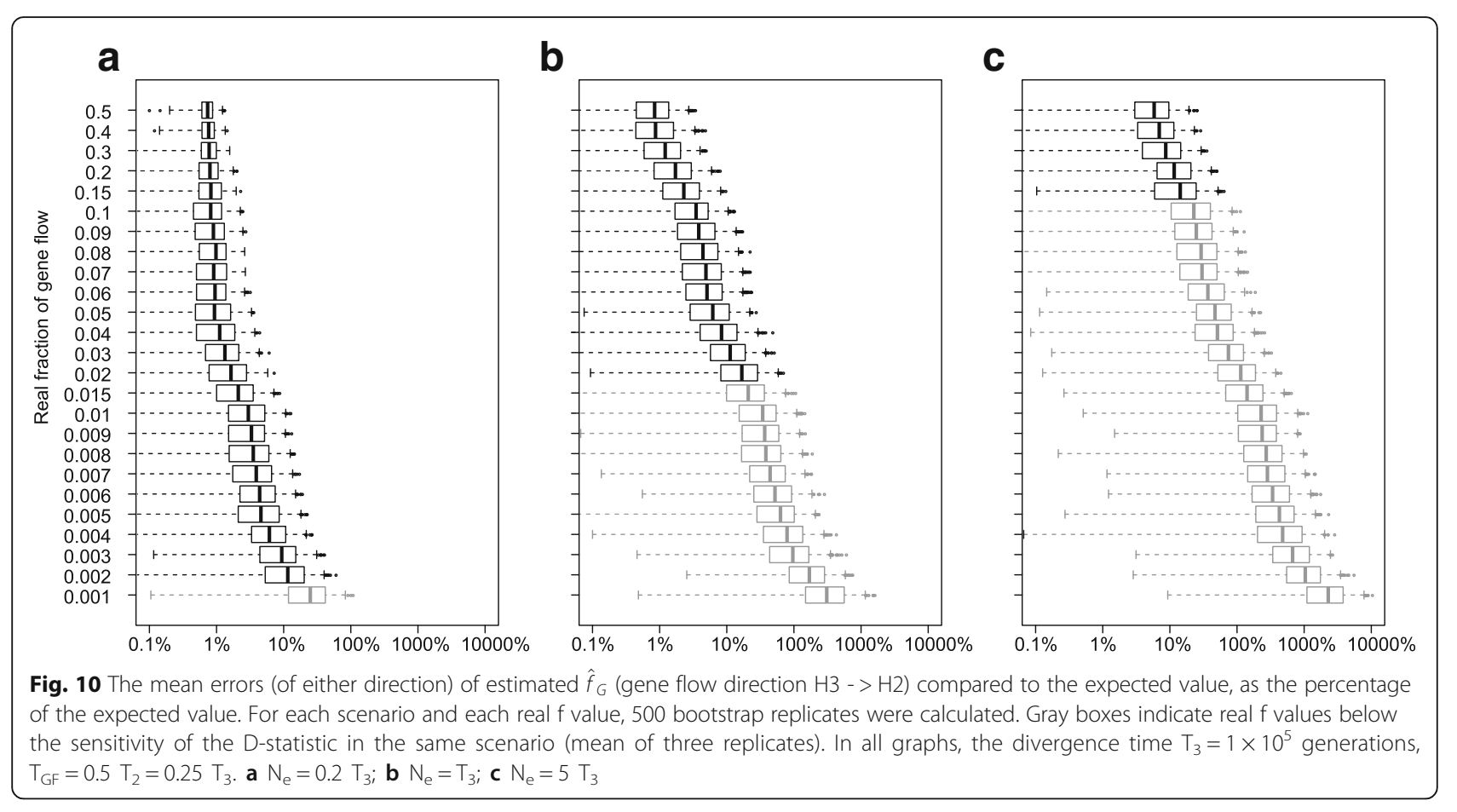

parameters. This is consistent with our results in previous sections where the D-statistic is less sensitive to $\mathrm{H} 2->\mathrm{H} 3$ gene flow. In extreme cases with large relative population sizes (Fig. 11i), f values between 0.15 and 0.5 were rarely distinguishable with statistical significance.

The effects of relative divergence and introgression times on robustness of the f-statistics is also similar to that on sensitivity of the D-statistic (data not shown). A larger range of real $\mathrm{f}$ values are undistinguishable significantly in datasets with older $\mathrm{H} 1-\mathrm{H} 2$ divergence and introgression, for both $\hat{f}_{G}$ and $\hat{f}_{\text {hom }}$ in the gene flow direction $\mathrm{H} 3->\mathrm{H} 2$. On the other hand, for the direction $\mathrm{H} 2->\mathrm{H} 3$ the situation is more complicated, with recent divergence causing the most uncertainty and intermediate divergence causing the least.

\section{Diploid data}

In studies based on biological samples, the final genomic sequence is often the consensus of two copies from a diploid organism. The two genome copies from the gene flow recipient population may have different sets of introgressed loci, but our model does not allow explicit estimation of how much the overlap may be. Instead, we simulated two extreme conditions: the two genome copies have either the exact same set of loci affected by gene flow, or independently drawn sets of loci. A realistic expectation lies between these two conditions.

We found that while the effect of ploidy on the sensitivity (as measured by MF80) of the analysis is not significant $(p=0.580$ for $\mathrm{H} 3->\mathrm{H} 2$, and $p=0.537$ for $\mathrm{H} 2->\mathrm{H} 3$ gene flow), it is significant for $\mathrm{H} 3->\mathrm{H} 2$ $(p=0.002)$ as well as for $\mathrm{H} 2->\mathrm{H} 3(p=0.035)$ when MF80 is log-transformed. Figure 12 shows the detailed effects of parameters and ploidy on sensitivity. It is evident that diploid data with independent sets of introgressed loci (blue dots) showed considerably increased MF80 values, but only under low relative population size. For datasets with a relative population size of 0.2 (lowest), the sensitivity increased from lower than 0.01 to between 0.01 and 0.05 , reaching 0.1 in some cases of $\mathrm{H} 2->\mathrm{H} 3$ gene flow. Note that this does not affect datasets with the same set of introgressed loci in both genome copies (green dots).

\section{Discussion \\ Relative population size as the key factor in parameter space}

The D-statistic was invented to analyze gene flow between Neanderthals and anatomically modern humans [14]. While it is suitable for both sequence data and population-wide allele frequency data [15], the Dstatistic is more often being used on sequences collected from closely-related taxa. Our research explored the parameter space of the phylogeny and demographics of the studied taxa, and determined how these parameters affect the sensitivity of the Dstatistic. Sensitivity is described with MF80, the minimal fraction of genome affected by gene flow to produce significant D-statistics in $80 \%$ of permutations. 


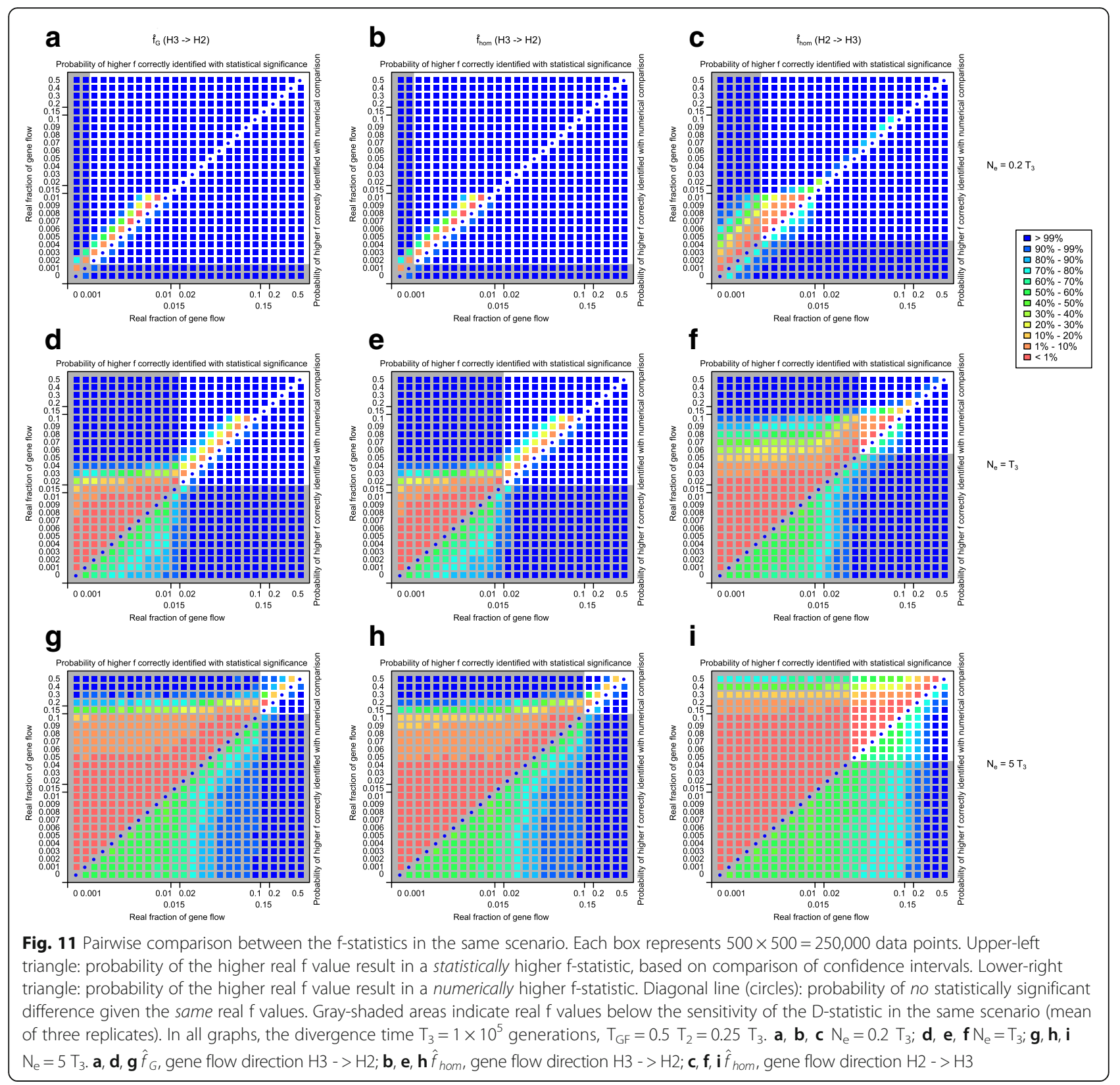

An MF80 of 0.01 means that the D-statistic can detect a $1 \%$ gene flow.

Our study marks one of the first attempts to explore the parameter space in which the D-statistic is reasonably sensitive. We have shown that the relative population size, which is the ratio of population size and divergence time in generations, is the most important factor on the sensitivity of the D-statistic. Indeed, the "coalescence time unit" as a measure of branch length is the reciprocal of the relative population size, and the probability of gene tree differs from the species tree in a three-species tree is $(2 / 3) \mathrm{e}^{-t}$ where $t$ is the number of coalescence time units $[28,29]$. Similarly, the proportion
$\mathrm{T}_{2} / \mathrm{T}_{3}$ and $\mathrm{T}_{\mathrm{gf}} / \mathrm{T}_{3}$ affects sensitivity through the lengths of branches separating the divergence and gene flow events. Branches short in coalescent time units are likely to produce ILS which add to both ABBA and BABA counts, diluting information for the D-statistic [1]. Our usage of relative population size describes how large the population size is compared to the entire history of the (H1, H2, H3) complex, in contrast to the coalescent time unit, which is used to measure the length of individual branches. In case of changing population sizes, the harmonic mean is commonly used [41]; future research may focus on obtaining a mean population size of multiple diverged populations or species. 

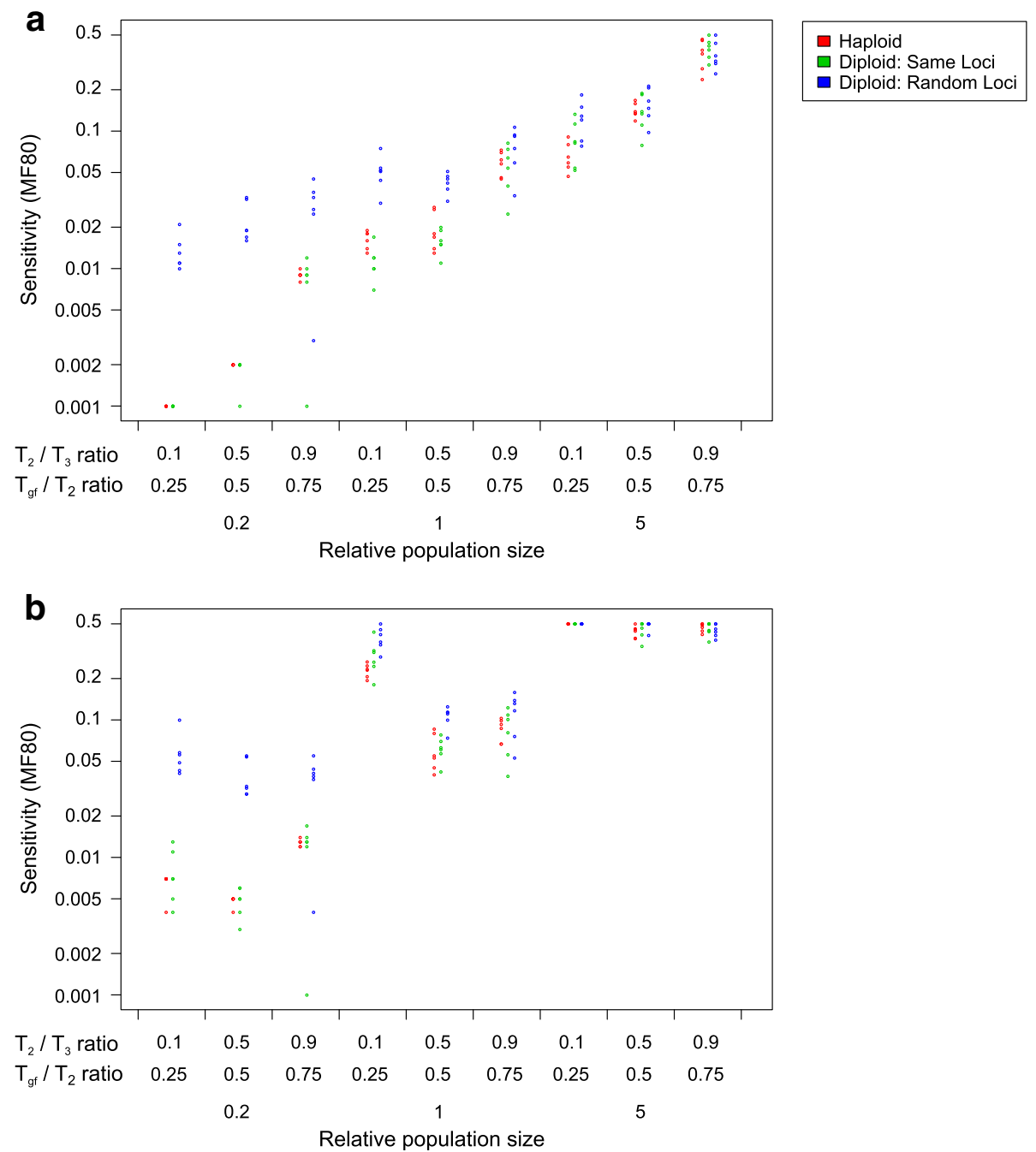

Fig. 12 Comparison of sensitivity of the D-statistic in haploid and diploid data. "Same loci" indicates two genome copies share the same set of introgrossed loci, while "random loci" indicates two genome copies have independently drawn sets of introgressed loci. The direction of gene flow is a $\mathrm{H} 3->\mathrm{H}_{2} ; \mathbf{b} \mathrm{H} 2->\mathrm{H} 3$

Sequence divergence, or genetic distance is not a crucial factor on the D-statistic's sensitivity alone, at least within a $0.2 \%$ to $20 \%$ range. The D-statistic analysis has been applied to biological questions with sequence divergences as low as $0.3 \%$ [14] and as high as about $5 \%[26,27]$. The analyses show that within reasonable range, sequence divergence is only a minor concern. A caveat, however, exists in the form of non-substitution mutations. Long-term evolution can accumulate mutations such as insertions, deletions and duplications in the genomes, causing incorrect mapping and alignment, which may affect the gene flow analysis by aligning non-homologous sites and producing artefactual ABBA or BABA sites. Therefore, high-coverage genomes and good alignment tools are essential for such studies.
We scaled both branch lengths and population sizes with the reciprocal of the substitution/mutation rate $\mu$, as it does not affect gene trees (branch lengths measured in substitutions per nucleotide) in a neutral model. This allows the interpretation of our results to be applicable to diverse organisms; humans have a rate of 1.3 to $2.5 \times$ $10^{-8}$ mutations per nucleotide per generation [42, 43], while in Heliconius butterflies the rate is approximately 2 to $3 \times 10^{-9}$ mutations per nucleotide per generation $[18,44,45]$. The relative population size, being a ratio of two parameters both scaled with $1 / \mu$, is completely independent from mutation rate in our model.

In the Neanderthal-human analysis, the relative population size is about 0.5 assuming $\mathrm{Ne}=10,000$ and $\mathrm{T}_{3}=20,000$ generations [14]. For the Heliconius butterflies, $\mathrm{Ne}=0.5$ to 2 million and $\mathrm{T}_{3}=8,000,000$ 
generations give a relative population size of 0.06 to 0.25 [46]. A study on gibbons gave $\mathrm{Ne}=$ circa 100,000 , generation time of ten years and $\mathrm{T}_{3}$ of circa 5 million years, so the relative population size is 0.2 [47]. These values all fall into a reasonable range in our study. In a study of gene flow among equids, $\mathrm{Ne}=200,000$, generation time of eight years and divergence time at some 2 million years, giving a relative population size of 0.8 [17]. While this is higher than the previously mentioned studies, strong gene flow over $10 \%$ of the genome can still be detected by the D-statistic analyses. Finally, in a study on dogs and wolves, the ancestral $\mathrm{Ne}$ was estimated at 35000 , generation time of three years and a dog-wolf divergence of circa 15,000 years, resulting in a relative population size of 7 [48]; however, bottlenecks during domestication could have reduced the actual effective population size and consequently incomplete lineage sorting. As a rule, when the relative population size is 0.5 or less, the D-statistic appears to be reliable. On groups with higher population sizes, alternative methods may be required to correctly identify inter-population or inter-species hybridization, using multiple samples within each population. However, studies that find all negative results for the D-statistic may choose not to include these findings in the published manuscript.

\section{Effects (or lack of effects) of outgroup distance and loci number}

Other parameters, such as outgroup distance and loci number, were also explored on their effect on the statistical analysis. A distant outgroup has been shown to, for example, reduce accuracy of phylogenetic rooting [49]. Counterintuitively, the distance between the outgroup and the ingroups within reasonable range seems not to be relevant to the sensitivity of the D-statistic as well as the errors of the f-statistics. The determining of ancestral allele in ABBA or BABA sites is based on the outgroup. It might be expected that a distant outgroup would cause more false positives and negatives in identifying such sites due to multiple substitutions (randomization), reducing the efficiency of the Dstatistic to detect gene flow. Simulating multiple substitution is possible with the program INDELible, because it evolves DNA sequences with each mutation being independently assigned. While the concern about alignment and mapping artifacts with a very distant outgroup still exist, it is a reassurance that the D-statistic analysis can be used even when a closely related species as an outgroup cannot be found.

The D-statistic is also shown to be more sensitive in a large number of smaller loci, given a constant size of analyzed genome. The most likely reason is a lower sampling error; even without gene flow, a single locus can favor ABBA or BABA sites due to incomplete lineage sorting. A larger number of loci means that the number ABBA-favoring and BABA-favoring loci are more similar to each other.

While we did not use a complex recombination model, loci number can be seen as a proxy of recombination rate. A higher recombination rate will break up linkage more often, which leads to an increase of locus number. Based on our results, when other conditions are similar, taxa with higher recombination rates are more sensitive to the D-statistic. Furthermore, given a constant rate of recombination, longer divergence time between taxa means more recombination events, thus increasing sensitivity because of reduced locus size; as we have shown in a previous section, genetic distance alone does not reduce sensitivity. Our main simulation scheme provides $20 \mathrm{~kb}$ loci, while the shortest loci are $10 \mathrm{~kb}$. Further reduction of length (thus increasing the number of independent loci) is constrained by computation time limitations. In biological datasets, if the loci are even shorter, one can expect even better sensitivity of the D-statistic compared with our results. In the future, backward simulations based on coalescence algorithms, such as msprime [50], can be employed to further pinpoint the effects of locus size and recombination rate.

\section{Direction of gene flow}

The D-statistic was developed to detect gene flow in both directions, i.e. $\mathrm{H} 2$ - > H3 and $\mathrm{H} 3->\mathrm{H} 2$. In the studies on Neanderthal and human genomes, Neanderthals are extinct and multiple non-African modern human populations were used, therefore it is reasonable to claim that the direction is from Neanderthal to modern humans $[14,15]$. However, with studies where all sampled taxa are extant and only one sample is available for each of the four taxa, the D-statistic alone cannot determine the direction of gene flow. A five-taxon statistic known as D-FOIL is able to determine direction of gene flow in some situations [51].

We have observed that, other parameters being identical, H3 - > H2 gene flow is easier to detect than $\mathrm{H} 2->$ $\mathrm{H} 3$ gene flow. A mutation that produces ABBA sites under $\mathrm{H} 3$ - > H2 gene flow must occur after $\mathrm{H} 3$ diverged from $\mathrm{H} 1 / \mathrm{H} 2\left(\mathrm{~T}_{3}\right)$ and before the gene flow $\left(\mathrm{T}_{\mathrm{GF}}\right)$; but such mutation under a $\mathrm{H} 2$ - > H3 gene flow must occur after $\mathrm{H} 2$ diverged from $\mathrm{H} 1\left(\mathrm{~T}_{2}\right)$ and before the gene flow $\left(\mathrm{T}_{\mathrm{GF}}\right)$. The former timespan is longer than the latter under the same demographic scenario; therefore, when the $\mathrm{f}$ is equal, $\mathrm{H} 3->\mathrm{H} 2$ gene flow produces more ABBA sites than $\mathrm{H} 2->\mathrm{H} 3$, making it easier to be detected with the D-statistic.

There is also an interesting finding regarding the direction of gene flow: more recent divergence between $\mathrm{H} 1$ and $\mathrm{H} 2$ hinders the detection of $\mathrm{H} 2$ - > $\mathrm{H} 3$ gene flow but helps the detection of $\mathrm{H} 3->\mathrm{H} 2$ gene flow. The former 
can be explained by that the timespan between $T_{2}$ and $\mathrm{T}_{\mathrm{g}}$, required for ABBA sites under $\mathrm{H} 2->\mathrm{H} 3$, is reduced when $\mathrm{T}_{2}$ is smaller. The latter may be explained by that more recent $\mathrm{H} 1-\mathrm{H} 2$ divergence means their (pre-introgression) sequences are more similar, providing a clearer background for the introgressed sites to be detected.

\section{False positives}

False positives are also a potential problem for the Dstatistic. While only one simulated dataset in a total of 789 has a $|Z|>3,45$ out of the these datasets in the main simulation scheme have $|Z|>1.96$, which would be significant had we set the significance level to be $p<$ 0.05 ; this is consistent with the proportion of such datasets (5.7\%). There are two main sources of false positives in the D-statistic. One is loci sampling error. ILS produce gene trees that group $\mathrm{H} 1$ and $\mathrm{H} 3$ together (favoring BABA sites) at the same rate as gene trees that group $\mathrm{H} 2$ and $\mathrm{H} 3$ together (favoring ABBA sites), which theoretically cancel out with each other. However, as the number of sampled loci is finite, the two types of gene trees may have different frequencies by chance, causing BABA and ABBA site number to be unbalanced. The other source of error is that the sequence from $\mathrm{H} 1$ being more or less distant than $\mathrm{H} 2$ from the $\mathrm{H} 1-\mathrm{H} 2$ common ancestor. This can be caused by a different evolutionary rate or sequencing error, the latter of which have been analytically tested by [15] for one specific set of parameter values.

To date, there is no simulation-based study on false positives of the D-statistic from either source, nor analysis on the interaction of them and other factors such as population size. This is possibly due to the fact that specificity tests, i.e. tests of false positive rates, require independent replicate datasets on which the D-statistic would be calculated under no gene flow. If all 135 parameter combinations of our main data scheme are simulated 500 times in parallel, the total running time would be estimated as 4.5 years. Future studies may focus on finding methods of permutation and subsampling so that independent or almost independent datasets can be generated within limited computation power, on which false positives can be studied.

\section{Usage of the f-statistics}

Our result showed that both $\hat{f}_{G}$ and $\hat{f}_{\text {hom }}$ are largely linearly correlated with the real fraction of gene flow, $\mathrm{f}$, but the usage of them to estimate $\mathrm{f}$ is hindered by parameters that are often unknown. These include the direction of gene flow and the $\mathrm{T}_{\mathrm{gf}} / \mathrm{T}_{3}$ ratio - the relative chronologic placement of the gene flow event. Sampling error due to finite number of loci can also introduce uncertainty, particularly when the fraction of gene flow is small. We conclude that the f-statistics cannot be used to reliably estimate the true fraction of gene flow without polymorphism data from a larger number of individuals in each population, or reliable estimates of divergence and introgression times as well as population sizes.

The linearity between the real $\mathrm{f}$ and $\mathrm{f}$-statistics can be exploited to compare the extent of introgression between different genomic regions with the same set of taxa, or taxa that have similar divergence times and population sizes. Our results showed that within the sensitivity of the D-statistic (which means that gene flow events can be detected at the first place), the random error of the f-statistics are moderate; $a \pm 20 \%$ error must be taken into account. However, a higher real $\mathrm{f}$ may not be statistically significant especially when the $f$ values are just above the D-statistic's sensitivity. It is common for a higher real $f$ to always report a numerically higher f-statistic but seldom statistically significant. Therefore, when comparing the f-statistics resulting from multiple tests under the same evolutionary scenario, it is preferred to choose numerical comparison under difficult conditions (large relative population size, early divergence/introgression and small difference between gene flow fractions), and to choose statistical comparison otherwise.

A few other f-statistic applications exist, but some of them require more sampled lineages than three ingroups and one outgroup; an example is $\mathrm{f}_{4}$-ratio estimation [31]. Others require population data, in which the allele frequencies from different populations are used, such as $f_{2}$ and $\mathrm{f}_{3}$ statistics [38] as well as $\hat{f}_{d}$ [22]. Here we focused on the situation that only one or two sequences are sampled from each taxon, and four taxa (three ingroups and one outgroups) are sampled; our conclusion is that, in such cases, $\hat{f}_{G}$ and $\hat{f}_{\text {hom }}$ are not very reliable estimator of actual fraction of gene flow, $\mathrm{f}$, except in special cases when population is very small and the time of gene flow is known.

\section{Diploid data}

We have shown that the sensitivity from diploid sequences where the two genome copies have different introgressed loci can be worse than haploid sequences or diploid sequences with the same set of introgressed loci, especially when the sensitivity is good at the first place (low relative population size). It is expected that, the more recent the gene flow happens, the more likely that two genome copies would have different set of introgressed loci. The reason for this is that alleles from recent migrants may not be fixed or lost yet, and segregate in the recipient population leading to heterozygous sites. When the heterozygous sites were removed in 
constructing a consensus sequence, valuable data to estimate gene flow were also lost. Therefore, our suggestion is that haploid sequence (acquired by sequencing haplotypes, or randomly assign one nucleotide for heterozygote sites) instead of consensus sequence from diploid samples should be used, when gene flow is recent and relative population size is small; otherwise consensus from diploid data is sufficient.

Still, our model does not explicitly predict how many introgressed sites are shared by the two genome copies, but analyzed two extreme situations and interpolated. In the future, it is possible that a more sophisticated coalescent-based model can be used to further investigate the effect of diploid data on the D-statistic.

Potential gene flow from an extinct or unsampled lineage Another possibility is that the gene flow originates from a lineage that is extinct or unsampled, commonly referred to as a "ghost lineage." Figure 13 shows three possible origins of such a lineage. When the "ghost" diverged after $\mathrm{H} 3$ did (Fig. 13a), the event cannot be detected by the $\mathrm{D}$-statistic, as a single mutation on the tree of an introgressed loci cannot produce either ABBA or BABA patterns. When the "ghost" is a sister species of $\mathrm{H} 3$ (Fig. 13b), the situation is identical to gene flow from $\mathrm{H} 3$ at the time when $\mathrm{H} 3$ and "ghost" diverged; any mutation occurring on the bolded branch can produce a species-tree-discordant site pattern. However, when the ghost diverged before H3 did (Fig. 13c), the situation is more complicated. A mutation occurring on the bold branch would have descendants in $\mathrm{H} 1, \mathrm{H} 2$ and $\mathrm{H} 3$, and if then the locus in $\mathrm{H} 2$ is replaced by a plesiomorphy from the ghost lineage, the site pattern will be "BABA," just like a gene flow from $\mathrm{H3}$ to $\mathrm{H1}$. Durand et al. [15] calculated the expected D-statistic during such a situation, but using only four taxa there is no method to differentiate such a "ghost introgression" from a gene flow from H3. Biologists would need to sample a larger range of species to determine where an introgression lineage come from.

\section{Conclusion}

In this study, we have shown that the D-statistic is more sensitive in detecting gene flow events when a) the population size to divergence ratio is small, b) gene flow is recent, and c) in the direction of $\mathrm{H} 3->\mathrm{H} 2$ (compared to $\mathrm{H} 2->\mathrm{H} 3$ ), and d) the data contains larger number of independent loci. On the other hand, the D-statistic is less sensitive to different levels of sequence divergence among ingroups or between ingroups and the outgroup. We have established the reliability of the D-statistic under a large range of parameter space. The f-statistics, while linearly correlated with the fraction of genome affected by gene flow, is not reliable for most

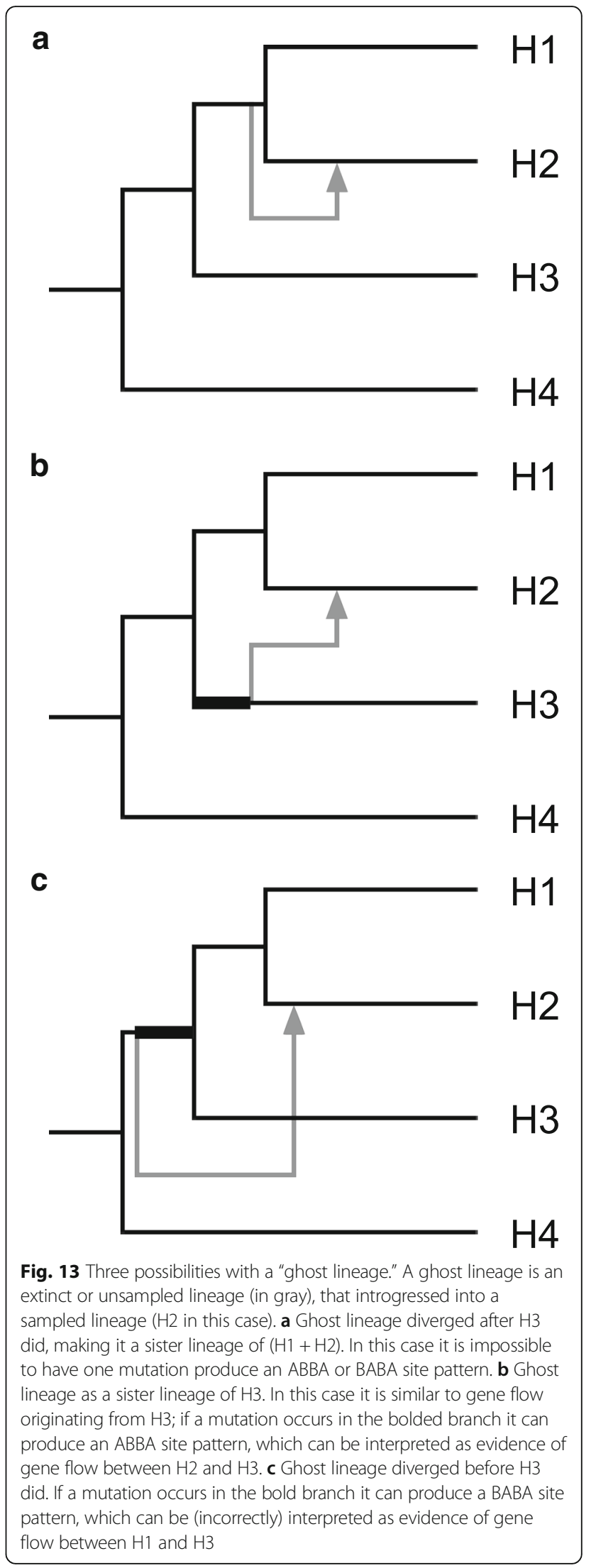


applications with individual (rather than population) samples, because its dependence on too many parameters, such as the time of gene flow event which can be difficult to accurately estimate; however it can be used to compare amount of introgression in the same demographic scenario. We have established that, as a rule of thumb, under a population size that equals or less than half of the number of generations since divergence of all tested species, the D-statistic is a sensitive method to detect gene flow.

\section{Additional files}

Additional file 1: Sensitivity and input parameters (continued) Description: The relationship of sensitivity as measured with MF80, the minimal fraction of gene flow that produces over $80 \%$ significant Dstatistics, and various input parameters: A. the ratio of divergence times, $T_{2}$ and $T_{3} ; B$. the ratio of time of gene flow $\left(T_{g f}\right)$ and $T_{2}$. Red points represent gene flow from $\mathrm{H} 3$ to $\mathrm{H} 2$, and green points represent gene flow from $\mathrm{H} 2$ to $\mathrm{H} 3$. (PDF $150 \mathrm{~kb}$ )

Additional file 2: Detailed results from each dataset. Description: Input parameters, sensitivity, significance information and linear regression of the f-statistics, from all datasets in three simulation schemes. (XLSX 214 kb)

\section{Acknowledgements}

We thank Dr. Vikas Kumar, Fritjof Lammers and Dr. Robert O'Hara for their valuable input and help during the analysis and writing of the manuscript.

\section{Funding}

This study was supported by the Leibniz Society.

\section{Availability of data and materials}

The datasets generated and/or analysed during the current study are not publicly available, due to all data being simulated with publically available programs and cannot be meaningfully archived.

\section{Authors' contributions}

YZ conducted the simulation and statistical analysis under AJ's direction, both authors contributed to the writing and the interpretation of the data. Both authors have read and approved the final version of the manuscript.

\section{Ethics approval and consent to participate}

Not Applicable

\section{Consent for publication \\ Not Applicable}

\section{Competing interests}

The authors declare that they have no competing interests.

Additional Note: Yichen Zheng's current address is Institut für Genetik,

Universität zu Köln, 50674, Cologne, Germany.

\section{Publisher's Note}

Springer Nature remains neutral with regard to jurisdictional claims in published maps and institutional affiliations.

Received: 26 April 2017 Accepted: 18 December 2017

Published online: 08 January 2018

\section{References}

1. Maddison WP. Gene trees in species trees. Syst Biol. 1997;46(3):523-36.

2. Avise JC, Neigel JE, Arnold J. Demographic influences on mitochondrial DNA lineage survivorship in animal populations. J Mol Evol. 1984;20(2):99-105.

3. Wolf YI, Rogozin IB, Grishin NV, Koonin EV. Genome trees and the tree of life. Trends Genet. 2002;18(9):472-9.
4. Carstens BC, Knowles LL. Estimating species phylogeny from gene-tree probabilities despite incomplete lineage sorting: an example from Melanoplus grasshoppers. Syst Biol. 2007:56(3):400-11.

5. Kubatko LS, Carstens BC, Knowles LL. STEM: species tree estimation using maximum likelihood for gene trees under coalescence. Bioinformatics. 2009; 25(7):971-3.

6. Maddison WP, Knowles LL. Inferring phylogeny despite incomplete lineage sorting. Syst Biol. 2006:55(1):21-30.

7. Mirarab S, Reaz R, Bayzid MS, Zimmermann T, Swenson MS, Warnow T. ASTRAL: genome-scale coalescent-based species tree estimation. Bioinformatics. 2014;30(17):i541-8.

8. Nakhleh L. Computational approaches to species phylogeny inference and gene tree reconciliation. Trends Ecol Evol. 2013;28(12):719-28.

9. Rieseberg LH, Morefield JD. Character expression, phylogenetic reconstruction, and the detection of reticulate evolution. Experimental and molecular approaches to plant biosystematics. 1995;53:333-54.

10. Sang T, Zhong Y. Testing hybridization hypotheses based on incongruent gene trees. Syst Biol. 2000;49(3):422-34

11. Than C, Ruths D, Nakhleh L. PhyloNet: a software package for analyzing and reconstructing reticulate evolutionary relationships. BMC Bioinformatics. 2008;9(1):1.

12. Yu Y, Dong J, Liu KJ, Nakhleh L. Maximum likelihood inference of reticulate evolutionary histories. Proc Nat Acad Sci USA. 2014;111(46): 16448-53.

13. Hobolth A, Christensen OF, Mailund T, Schierup MH. Genomic relationships and speciation times of human, chimpanzee, and gorilla inferred from a coalescent hidden Markov model. PLoS Genet. 2007;3(2):e7.

14. Green RE, Krause J, Briggs AW, Maricic T, Stenzel U, Kircher M, Patterson N, Li H, Zhai W, Fritz MH, Hansen NF. A draft sequence of the Neandertal genome. Science. 2010;328(5979):710-22.

15. Durand EY, Patterson N, Reich D, Slatkin M. Testing for ancient admixture between closely related populations. Mol Biol Evol. 2011;28(8):2239-52.

16. Kumar V, Lammers F, Bidon T, Pfenninger M, Kolter L, Nilsson MA, Janke A. The evolutionary history of bears is shaped by gene flow across species. Sci Rep. 2017;7:46487

17. Jónsson $H$, Schubert $M$, Seguin-Orlando A, Ginolhac A, Petersen $L$, Fumagall M, Albrechtsen A, Petersen B, Korneliussen TS, Vilstrup JT, Lear T. Speciation with gene flow in equids despite extensive chromosomal plasticity. Proc Nat Acad Sci USA. 2014;111(52):18655-60.

18. Dasmahapatra KK, Walters JR, Briscoe AD, Davey JW, Whibley A, Nadeau NJ, Zimin AV, Hughes DS, Ferguson LC, Martin SH, Salazar C, et al. Butterfly genome reveals promiscuous exchange of mimicry adaptations among species. Nature. 2012;487(7405):94-8.

19. Escudero M, Eaton DA, Hahn M, Hipp AL. Genotyping-by-sequencing as a tool to infer phylogeny and ancestral hybridization: a case study in Carex (Cyperaceae). Mol Phylogenet Evol. 2014;79:359-67.

20. Owens GL, Baute GJ, Rieseberg LH. Revisiting a classic case of introgression: hybridization and gene flow in Californian sunflowers. Mol Ecol. 2016;25(11): 2630-43.

21. Martin MD, Vieira FG, Ho SY, Wales N, Schubert M, Seguin-Orlando A, Ristaino JB, Gilbert MT. Genomic characterization of a south American Phytophthora hybrid mandates reassessment of the geographic origins of Phytophthora infestans. Mol Biol Evol. 2016;33(2):478-91.

22. Martin SH, Davey JW, Jiggins CD. Evaluating the use of ABBA-BABA statistics to locate introgressed loci. Mol Biol Evol. 2015:32(1):244-57.

23. Martin SH, Dasmahapatra KK, Nadeau NJ, Salazar C, Walters JR, Simpson F, Blaxter M, Manica A, Mallet J, Jiggins CD. Genome-wide evidence for speciation with gene flow in Heliconius butterflies. Genome Res. 2013; 23(11):1817-28

24. Kronforst MR. Gene flow persists millions of years after speciation in Heliconius butterflies. BMC Evol Biol. 2008;8(1):1.

25. Martin SH, Eriksson A, Kozak KM, Manica A, Jiggins CD. Speciation in Heliconius Butterflies: Minimal Contact Followed by Millions of Generations of Hybridisation. bioRxiv. 2015:015800.

26. Fontaine MC, Pease JB, Steele A, Waterhouse RM, Neafsey DE, Sharakhov IV, Jiang $X$, Hall AB, Catteruccia F, Kakani E, Mitchell SN. Extensive introgression in a malaria vector species complex revealed by phylogenomics. Science. 2015:347(6217):1258524

27. Brandvain Y, Kenney AM, Flagel L, Coop G, Sweigart AL. Speciation and introgression between Mimulus nasutus and Mimulus guttatus. PLoS Genet. 2014;10(6):e1004410. 
28. Nei M. Molecular evolutionary genetics: Columbia university press; 1987.

29. Wakeley J. Coalescent theory. Roberts \& Company. 2009;

30. Efron B. Nonparametric estimates of standard error: the jackknife, the bootstrap and other methods. Biometrika. 198168(3):589-599.

31. Reich D, Thangaraj K, Patterson N, Price AL, Singh L. Reconstructing Indian population history. Nature. 2009;461(7263):489-94.

32. Mallo D, Martins LD, Posada D. Simphy: phylogenomic simulation of gene, locus, and species trees. Syst Biol. 2016;65(2):334-44.

33. Hudson RR. Properties of a neutral allele model with intragenic recombination. Theoretical Population. Biol. 1983;23(2):183-201.

34. Kingman JF. On the genealogy of large populations. J Appl Probab. 1982; 19(A):27-43.

35. Fletcher W, Yang Z. INDELible: a flexible simulator of biological sequence evolution. Mol Biol Evol. 2009;26(8):1879-88.

36. Iwagami M, Rajapakse RP, Paranagama W, Agatsuma T. Identities of two Paragonimus species from Sri Lanka inferred from molecular sequences. J Helminthology. 2003;77(3):239-45.

37. Largiader CR, Herger F, Lörtscher M, Scholl A. Assessment of natural and artificial propagation of the white-clawed crayfish (Austropotamobius pallipes species complex) in the alpine region with nuclear and mitochondrial markers. Mol Ecol. 2000;9(1):25-37.

38. Patterson N, Moorjani P, Luo Y, Mallick S, Rohland N, Zhan Y, Genschoreck T, Webster T, Reich D. Ancient admixture in human history. Genetics. 2012 192(3):1065-93.

39. Ellis PD. The essential guide to effect sizes: statistical power, meta-analysis, and the interpretation of research results: Cambridge University Press; 2010.

40. Gibbs RA, Weinstock GM, Metzker ML, Muzny DM, Sodergren EJ, Scherer S, Scott G, Steffen D, Worley KC, Burch PE, Okwuonu G. Genome sequence of the Brown Norway rat yields insights into mammalian evolution. Nature. 2004;428(6982):493-521.

41. Vucetich JA, Waite TA, Nunney L. Fluctuating population size and the ratio of effective to census population size. Evolution. 1997;51(6):2017-21.

42. Nachman MW. Crowell SL. Estimate of the mutation rate per nucleotide in humans. Genetics. 2000;156(1):297-304.

43. Lynch M. Evolution of the mutation rate. Trends Genet. 2010;26(8):345-52.

44. Keightley PD, Pinharanda A, Ness RW, Simpson F, Dasmahapatra KK, Mallet J, Davey JW, Jiggins CD. Estimation of the spontaneous mutation rate in Heliconius melpomene. Mol Biol Evol. 2015;32(1):239-43.

45. Martin SH, Möst M, Palmer WJ, Salazar C, McMillan WO, Jiggins FM, Jiggins CD. Natural selection and genetic diversity in the butterfly Heliconius melpomene. Genetics. 2016;203(1):525-41.

46. Pardo-Diaz C, Salazar C, Baxter SW, Merot C, Figueiredo-Ready W, Joron M, McMillan WO, Jiggins CD. Adaptive introgression across species boundaries in Heliconius butterflies. PLoS Genet. 2012:8(6):e1002752.

47. Carbone L, Harris RA, Gnerre S, Veeramah KR, Lorente-Galdos B, Huddleston J, Meyer TJ, Herrero J, Roos C, Aken B, Anaclerio F. Gibbon genome and the fast karyotype evolution of small apes. Nature. 2014;513(7517):195-201.

48. Freedman AH, Gronau I, Schweizer RM, Ortega-Del Vecchyo D, Han E, Silva PM, Galaverni M, Fan Z, Marx P, Lorente-Galdos B, Beale H. Genome sequencing highlights the dynamic early history of dogs. PLoS Genet. 2014; 10(1):e1004016.

49. Graham SW, Olmstead RG, Barrett SC. Rooting phylogenetic trees with distant outgroups: a case study from the commelinoid monocots. Mol Biol Evol. 2002;19(10):1769-81.

50. Kelleher J, Etheridge AM, McVean G. Efficient coalescent simulation and genealogical analysis for large sample sizes. PLoS Comp Biol. 2016;12(5): e1004842.

51. Pease JB, Hahn MW. Detection and polarization of introgression in a fivetaxon phylogeny. Syst Biol. 2015;64(4):651-62.

\section{Submit your next manuscript to BioMed Central and we will help you at every step:}

- We accept pre-submission inquiries

- Our selector tool helps you to find the most relevant journal

- We provide round the clock customer support

- Convenient online submission

- Thorough peer review

- Inclusion in PubMed and all major indexing services

- Maximum visibility for your research

Submit your manuscript at www.biomedcentral.com/submit
C Biomed Central 